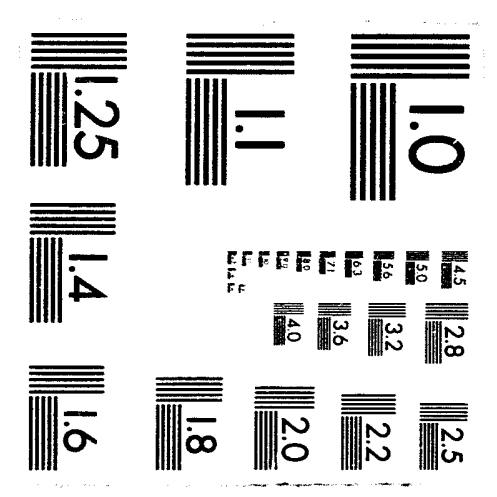



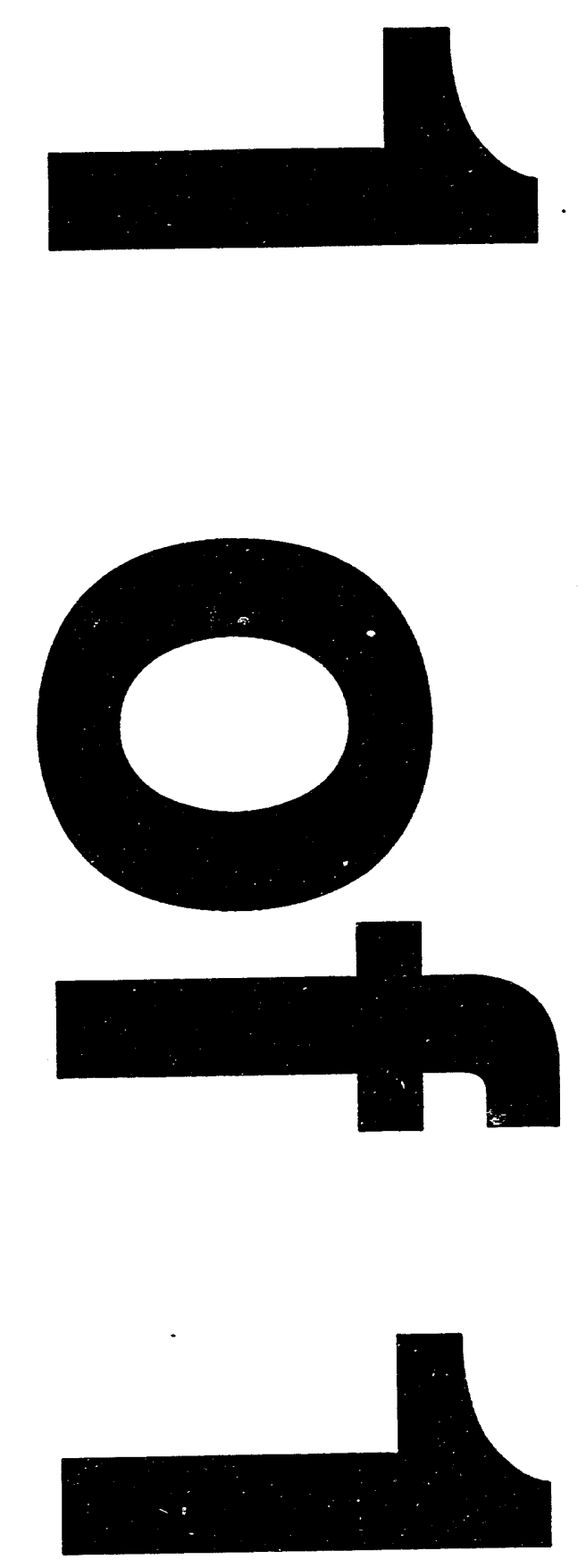
BNL-52417

UC-406

\section{HYDRODYNAMIC EFFECTS IN TANKS CONTAINING LAYERED LIQUIDS}

A. Veletsos, P. Shivakumar, and K. Bandyopadhyay

March 1994

Prepared for

OFFICE OF ENVIRONMENTÁL, RESTORATION AND WASTE MANAGEMENT DEPARTMENT OF ENERGY, WASHINGTON, D.C.

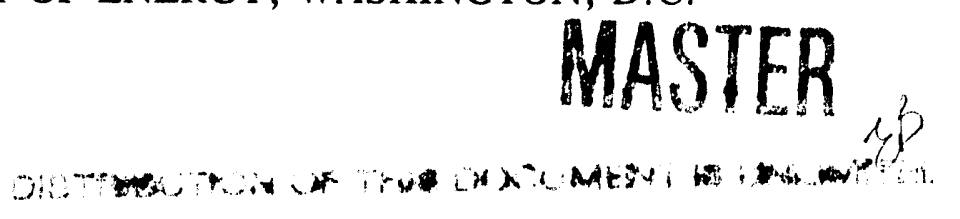




\begin{abstract}
As a supplement to a recently reported study, the hydrodynamic wall pressures and the associated tank forces induced by horizontal ground shaking in a rigid, vertical, circular cylindrical tank containing liquid layers of different thicknesses and mass densities are examined, and comprehensive numerical solutions are presented for twolayered and some three-layered systems which elucidate the underlying response mechanisms and the effects of the various parameters involved. Both the impulsive and convective actions are studied. Additionally, solutions are presented for multi-layered systems approximating a liquid with an exponential, continuous variation in density, and the interrelationship of the solutions for the continuous system and its discretized, layered approximation is discussed.
\end{abstract}




\section{TABLE OF CONTENTS}

Section

Page

ABSTRACT $\ldots \ldots \ldots \ldots \ldots \ldots \ldots \ldots \ldots \ldots \ldots$ ii

TABLE OF CONTENTS $\ldots \ldots \ldots \ldots \ldots \ldots \ldots$

LIST OF FIGURES $\ldots \ldots \ldots \ldots \ldots \ldots \ldots \ldots \ldots \ldots \ldots$ vii

LIST OF TABLES $\ldots \ldots \ldots \ldots \ldots \ldots \ldots \ldots \ldots \ldots$ viii

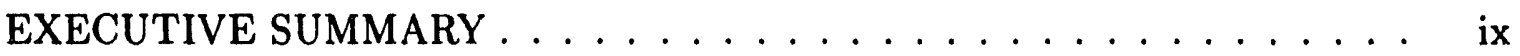

ACKNOWLEDGMENT $\ldots \ldots \ldots \ldots \ldots \ldots \ldots \ldots \ldots \ldots$ xi

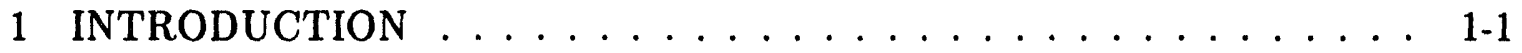

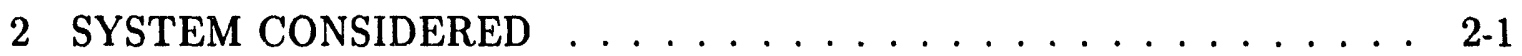

3 METHOD OF ANALYSIS . . . . . . . . . . . . . . 3-1

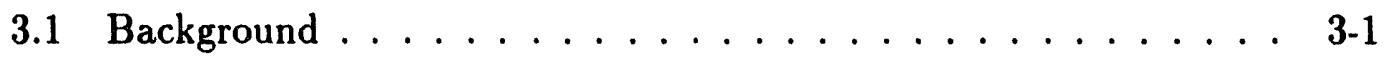

3.2 Hydrodynamic Pressures . . . . . . . . . . . . . . 3-3

3.2.1 Simplification of solution for impulsive pressures . . . . 3-4

3.2.2 Wall pressures. . . . . . . . . . . . . . 3-5

3.2.3 Specialized expressions for wall pressures. . . . . . . . . 3-5

3.3 Tank Forces . . . . . . . . . . . . . . . . . . . 3-6

3.3.1 Base shear . . . . . . . . . . . . . . . . 3-6

3.3.2 Moment above base . . . . . . . . . . . . . . . 3-7

3.3.3 Foundation moment . . . . . . . . . . . . 3-7

4 PRESENTATION AND ANALYSIS OF NUMERICAL SOLUTIONS . . 4-1

4.1 Hydrodynamic Wall Pressures . . . . . . . . . . . . . 4-1

4.1.1 Normalization of Wall Pressures . . . . . . . . . . 4-1

4.1.2 Representative Wall Pressures . . . . . . . . . . . 4-2

4.1.3 Wall Pressures for Two-Layered Systems . . . . . . . 4-4

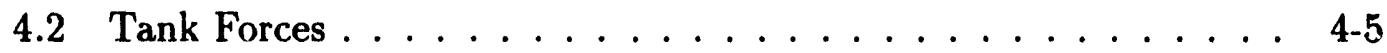


4.2.1 Two-layered systems . . . . . . . . . . . . 4-5

4.2.2 Three-layered systems . . . . . . . . . . . 4-6

4.3 Solutions for Continuous Systems . . . . . . . . . . . . 4-6

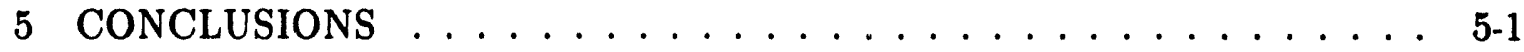

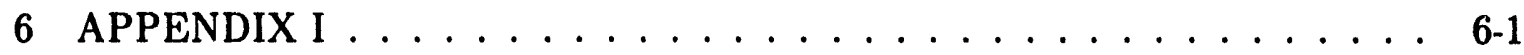

6.1 Derivation of Eq. $(23) \ldots \ldots$ 6-1 . . . . . . . . .

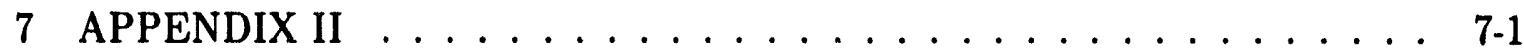

7.1 Derivation of Eq. $(51) \ldots \ldots \ldots$ 7-1 . . . . . . .

8 REFERENCES ....................... 8 . . . . .

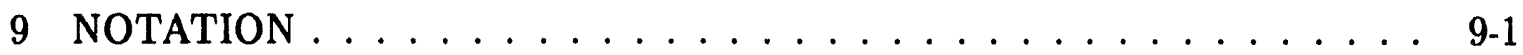




\section{LIST OF FIGURES}

$\begin{array}{lll}\text { Figure } & \text { Page }\end{array}$

2.1 System considered . . . . . . . . . . . . . . 2-2

4.1 Interrelationship of coefficients for impulsive and convective components of wall pressure for two-layered system with $H / R=1, H_{2} / H_{1}=1$ and $\rho_{2} / \rho_{1}=0.5 \ldots \ldots \ldots \ldots \ldots \ldots \ldots \ldots \ldots \ldots \ldots \ldots \ldots \ldots$

4.2 Interrelationship of coefficients for impulsive and convective components of wall pressure for three-layered system with $H / R=1, H_{1}=H_{2}=$ $H_{3}=H / 3$ and $\rho_{3} / \rho_{2} / \rho_{1}=1 / 2 / 3 \ldots \ldots \ldots \ldots \ldots$

4.3 Effect of $\rho_{2} / \rho_{1}$ on impulsive and fundamental convective pressure distributions for $H / R=1.0, H_{2} / H_{1}=1 \ldots \ldots \ldots \ldots$

4.4 Effect of $\rho_{2} / \rho_{1}$ on convective pressure coefficient $c_{1 n}(\eta)$ as $\rho_{2} / \rho_{1}$ tends to zero; system with $H / R=1 \ldots \ldots \ldots \ldots$

4.5 Impulsive and fundamental convective masses for two-layered systems with $H_{2} / H_{1}=1 \ldots \ldots \ldots \ldots \ldots \ldots \ldots$

4.6 Normalized values of coefficients for impulsive and fundamental convective component of base moment for two-layered systems with $H_{2} / H_{1}$ $=1 \ldots \ldots \ldots \ldots \ldots \ldots \ldots \ldots$

4.7 Normalized values of coefficients for impulsive and fundamental convective component of foundation moment for two-layered systems with $H_{2} / H_{1}=1 \ldots \ldots \ldots \ldots \ldots \ldots \ldots$

4.8 Convective masses for first two horizontal and two vertical modes of vibration of two-layered systems with $H_{2} / H_{1}=1 \ldots \ldots \ldots \ldots$

4.9 Comparison of wall pressure distributions for continuous system with $H / R=1$ and $\rho(1) / \rho_{o}=0.25$ with those of its layered approximations 


\section{LIST OF TABLES}

\section{Table}

4.1 Values of coefficients in expression for hydrodynamic wall pressure at selected sections of two-layered systems with different $\mathrm{H} / \mathrm{R}$ and $\mathrm{H}_{2} / \mathrm{H}_{1}$

4.2 Normalized values of effective masses in expression for base shear of two-layered systems with different $H / R$ and $H_{2} / H_{1} \ldots \ldots \ldots$. . . . . . 49

4.3 Normalized values of coefficients in expression for overturning moment at a section immediately above tank base of two-layered systems with different $H / R$ and $H_{2} / H_{1} \ldots \ldots \ldots \ldots \ldots$

4.4 Normalized values of coefficients in expression for foundation moment of two-layered systems with different $H / R$ and $H_{2} / H_{1} \ldots \ldots$

4.5 Normalized values of effective masses in expression for base shear of three-layered systems with different $H / R$ and $H_{1}=H_{2}=H_{3}=H / 3$.

4.6 Normalized valıes of coefficients in expression for overturning moment at a section immediately above tank base of three-layered systems with different $H / R$ and $H_{1}=H_{2}=H_{3}=H / 3 \ldots \ldots \ldots$

4.7 Normalized values of coefficients in expression for foundation moment of three-layered systems with different $H / R$ and $H_{1}=H_{2}=H_{3}=H / 3$

4.8 Normalized values of effective masses in expression for base shear of a continuous system with $H / R=1, \rho(1) / \rho_{o}=0.25$ and its $N$-layered

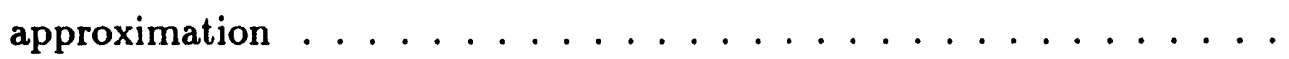

4.9 Normalized values of coefficients in expression for overturning moment at a section immediately above tank base for a continuous system with $H / R=1, \rho(1) / \rho_{o}=0.25$ and its $\mathrm{N}$-layered approximation . . . . .

4.10 Normalized values of coefficients in expression for foundation moment of a continuous system with $H / R=1, \rho(1) / \rho_{o}=0.25$ and its $\mathrm{N}$-layered

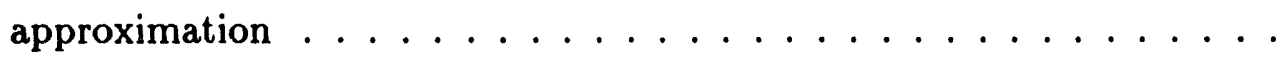




\section{EXECUTIVE SUMMARY}

The study reported herein is a sequel to the one described in BNL Report 52378, and it is motivated by the need for improved understanding of the response to earthquakes of waste-storage tanks in nuclear facilities. It deals with the hydrodynamic effects induced by horizontal ground shaking in rigid vertical circular cylindrical tanks containing an arbitrary number of uniform liquid layers of different thicknesses and densities.

Whereas the previous study dealt with the free vibrational characteristics of the systems and with the surface sloshing motions induced by ground shaking, the present study focuses on the evaluation of the corresponding wall pressures and tank forces. Both the impulsive and convective actions are examined. Comprehensive numerical solutions are presented for two-layered and three-layered systems which elucidate the underlying response mechanisms and the effects and relative importance of the various parameters involved. The results are compared with those computed on the assumption that the entire liquid acts as a rigid mass, and simple relations are established between the responses of layered and homogeneous systems and between the magnitudes of the impulsive and convective effects. Additionally, solutions are presented for multi-layered systems approximating a liquid with an exponential, continuous variation in density, and the interrelationship of the solutions for the continuous system and its discretized, layered approximation is discussed.

The principal conclusions of the study are as follows :

1. The response of an $N$-layered system may be expressed as the sum of an impulsive component and an infinite number of horizontal convective or sloshing components, each associated with $N$ vertical modes of vibration.

2. The $n$th vertical mode for each horizontal mode of vibration exhibits $(n-1)$ changes in sign. These changes are due to the in-phase or out-of-phase sloshing actions of the interfaces.

3. The impulsive pressure component is continuous and increases from zero at the top to a maximum at the base, whereas the convective pressure components are discontinuous at the layer interfaces, the magnitude of the discontinuity being 
a function of the tank proportions and of the relative densities and thicknesses of the layers.

4. When normalized with respect to the pressures computed on the assumption that the entire liquid acts as a rigid mass, the coefficients for the impulsive and all convective components of the hydrodynamic wall pressures add up to unity. The same is also true of the corresponding coefficients for base shear and base moments in the tank.

5. The impulsive component of response may be evaluated either as the difference between the response computed on the assumption that the entire liquid acts as a rigid mass and the sum of all convective components of response or, independently, without the prior evaluation of the convective effects.

6. For two-layered systems with ratios of mass densities in the range between 0.5 and unity, the base shear and base moments may be related simply to those obtained from well-established solutions for homogeneous systems.

7. The solutions for layered systems presented herein may also be used to accurately evaluate the responses of systems with arbitrary and continuous variations in liquid density. 


\section{ACKNOWLEDGMENT}

This study was carried out at Rice University in cooperation with Brookhaven National Laboratory (BNL). The authors are grateful to the Department of Energy Project Directors John Tseng, James Antizzo, Howard Eckert and Dinesh Gupta for supporting the study, and to Morris Reich of BNL for his understanding project management. Comments received from colleagues in BNL's Tank Seismic Expert Panel are also acknowledged with thanks. 


\section{SECTION 1}

\section{INTRODUCTION}

The study described herein is a sequel to the one reported recently [1] and is motivated by the need for improved understanding of the response to earthquakes of wastestorage tanks in nuclear facilities. It deals with the hydrodynamic effects induced by horizontal ground shaking in rigid vertical circular cylindrical tanks containing an arbitrary number of uniform liquid layers of different thicknesses and densities. The results are also of value in dynamic response studies of spent fuel reprocessing tanks.

In addition to the governing equations of motion for multi-layered systems, the previous study provided comprehensive numerical solutions for the free vibrational characteristics and the surface sloshing motions of systems with two and three layers. The present study deals with the evaluation of the corresponding wall pressures and tank

forces. Some attention is also given to the response of systems with a continuous variation in liquid density, and to the interrelationship of the solutions obtained for the continuous variation and its discretized, multi-layered representation.

The objectives are to elucidate the response mechanisms of the systems referred to, and to provide information and concepts with which the effects of the primary parameters may be evaluated rationally and conveniently for design purposes.

The response quantities examined include the hydrodynamic wall pressures, the associated base shears, and the bending moments at sections immediately above and below the tank base. Both the impulsive and convective actions are examined. The impulsive effects reflect the action of the part of the liquid that may be considered to move in synchronism with the tank wall as a rigidly attached mass, whereas the convective effects represent the action of the part of the liquid undergoing sloshing motions. The results are compared with those computed on the assumption that the entire liquid acts as a rigid mass, and simple relations are established between the responses of layered and homogeneous systems and between the magnitudes of the impulsive and convective effects.

The response of two-layered systems has been the subject of several recent studies by Tang and Chang $[2,3,4,5]$. The scope of these studies and their relatior ship to 
the authors' work have been identified in References 6,7 and 8 and are not repeated here. In addition to complementary numerical solutions for such systems, the present study provides information and interpretations which further clarify the underlying response mechanisms and the effects and relative importance of the numerous parameters involved. 


\section{SECTION 2}

\section{SYSTEM CONSIDERED}

The system investigated is shown in Fig. 2.1. It is a rigid, vertical, circular cylindrical tank of radius $R$ that is filled to a height $H$ with a multi-layered liquid, and is anchored to a rigid, horizontally oscillating base. The individual layers are considered to be uniform, but their densities and thicknesses may vary from one layer to the next. The liquid is assumed to be incompressible, irrotational and inviscid, and only linear actions are examined.

The liquid layers are numbered sequentially starting with 1 at the bottom layer and terminating with $N$ at the top layer. The common boundary to the $j$ th and $(j+1)$ th layers is designated as the $j$ th interface. The thickness and mass density of the $j$ th layer are denoted by $H_{j}$ and $\rho_{j}$, respectively, and the values of $\rho_{j}$ are considered to increase from top to bottom (i.e., decrease with increasing values of $j$ ). Points within îhe $j$ th layer are defined by the local cylindrical coordinate system, $r, \theta, z_{j}$, as shown in Fig. 2.1, with the origin of $z_{j}$ taken at the $(j-1)$ th interface.

The ground motion is considered to be uniform over the tank base and to be directed along the $\theta=0$ coordinate axis. The acceleration of the ground motion at any time $t$ is denoted by $\ddot{x}_{g}(t)$, and the corresponding velocity and displacement are denoted by $\dot{x}_{g}(t)$ and $x_{g}(t)$, respectively. 

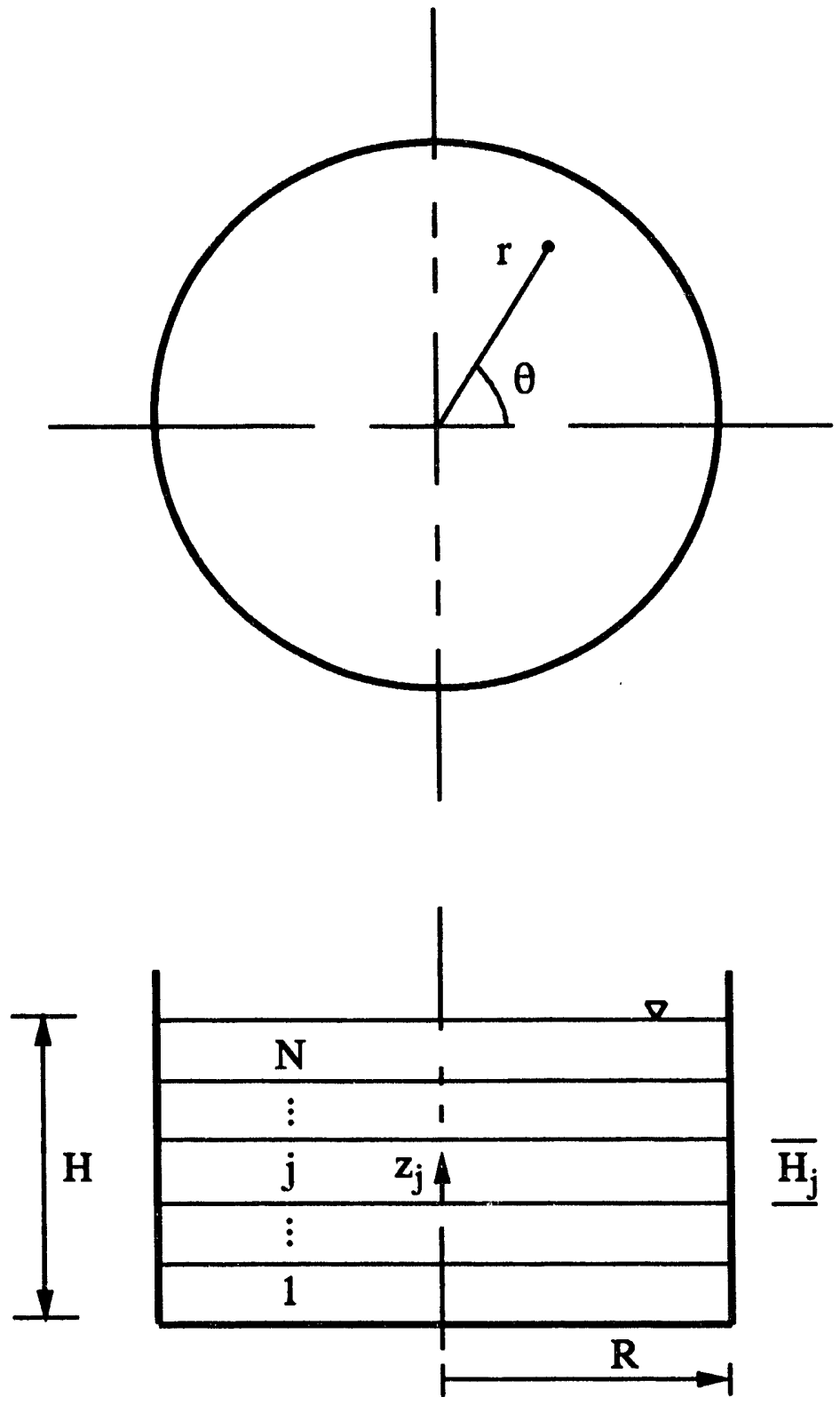

Figure 2.1 System considered 


\section{SECTION 3}

\section{METHOD OF ANALYSIS}

\subsection{Background}

The solutions presented herein are deduced from expressions presented in Reference 1, of which those needed in the following developments are summarized in this section.

The flow field in the $j$ th layer is specified by a velocity potential function, $\phi_{j}$, which satisfies Laplace's equation and is related to the corresponding hydrodynamic pressure, $p_{j}$, by

$$
p_{j}=\rho_{j} \frac{\partial \phi_{j}}{\partial t}
$$

The solution for $\phi_{j}$ is obtained by the superposition of two component solutions as

$$
\phi_{j}=-r \cos \theta \dot{x}_{g}(t)+\psi_{j}
$$

where

$$
\psi_{j}=-\sum_{m=1}^{\infty} \frac{R}{\lambda_{m}}\left[\frac{\dot{D}_{m, j}(t) \cosh \lambda_{m} \eta_{j}-\dot{D}_{m, j-1}(t) \cosh \lambda_{m}\left(\alpha_{j}-\eta_{j}\right)}{\sinh \lambda_{m} \alpha_{j}}\right] \frac{J_{1}\left(\lambda_{m} \xi\right)}{J_{1}\left(\lambda_{m}\right)} \cos \theta
$$

In the latter expression, $D_{m, j}(t)$ represents the instantaneous value of the vertical (sloshing) displacement of the liquid, when vibrating in its $m$ th horizontal natural mode, at a point along the wall located on the $j$ th interface and the $\theta=0$ axis; a dot superscript denotes differentiation with respect to time; $J_{1}(x)$ represents the Bessel function of the first kind of the variable $x$; and $\lambda_{m}$ represents the $m$ th root of the first derivative of $J_{1}(x)$. The first three of these roots are

$$
\lambda_{1}=1.841 \quad \lambda_{2}=5.331 \quad \lambda_{3}=8.536
$$

Additionally, $\alpha_{j}=H_{j} / R, \eta_{j}=z_{j} / R$ and $\xi=r / R$. The vector $\left\{D_{m}\right\}$ of the $N$ values of $D_{m, j}(t)$ are determined from the solution of the system of differential equations

$$
[\mathcal{A}]\left\{\ddot{D}_{m}\right\}+\frac{\lambda_{m} g}{R}[\mathcal{B}]\left\{D_{m}\right\}=-\epsilon_{m} \lambda_{m}\{s\} \ddot{x}_{g}(t)
$$

in which $[\mathcal{A}]$ is a tri-diagonal, symmetric matrix of size $N \times N ;[\mathcal{B}]$ is a diagonal matrix of the same size; $\{s\}$ is a vector of size $N$, the elements of which are the same as the corresponding diagonal elements of $[\mathcal{B}] ; g$ is the gravitational acceleration; and

$$
\epsilon_{m}=\frac{2}{\left(\lambda_{m}^{2}-1\right)}
$$


In Reference 1 , the matrices $[\mathcal{A}],[\mathcal{B}]$ and $\{s\}$ were denoted by $[A],[B]$ and $\{c\}$. The change is made so as to avoid confusion with symbols used in subsequent sections.

The solution of Eq. (5) may be expressed by modal superposition as

$$
\left\{D_{m}(t)\right\}=-R \sum_{n=1}^{N}\left\{d_{m n}\right\} \frac{A_{m n}(t)}{g}
$$

in which $\left\{d_{m n}\right\}$ are vectors of the dimensionless coefficients defined by

$$
\left\{d_{m n}\right\}=\epsilon_{m} \frac{\left\{\hat{D}_{m n}\right\}^{T}\{s\}}{\left\{\hat{D}_{m n}\right\}^{T}[\mathcal{B}]\left\{\hat{D}_{m n}\right\}}\left\{\hat{D}_{m n}\right\}
$$

$\left\{\hat{D}_{m n}\right\}$ is the vector of the maximum vertical or sloshing displacement amplitudes of the liquid at the layer interfaces when the sysiem is vibrating in its $m$ th horizontal and $n$th vertical natural mode; and $A_{m n}(t)$ is the instantaneous value of the corresponding modal pseudoacceleration of the system to the prescribed base motion. The latter function is given by

$$
A_{m n}(t)=\omega_{m n} \int_{0}^{t} \ddot{x}_{g}(\tau) \sin \left[\omega_{m n}(t-\tau)\right] d \tau
$$

wherein $\omega_{m n}$ is the circular frequency of the system for the mode of vibration considered. The vectors $\left\{\hat{D}_{m n}\right\}$ and the associated frequencies may be evaluated from Eq. (5) by equating its right-hand member to zero and solving the resulting eigenvalue problem, as previously indicated [1]. The frequency $\omega_{m n}$ may be conveniently expressed as

$$
\omega_{m n}=C_{m n} \sqrt{\frac{\lambda_{m} g}{R}}
$$

in which $C_{m n}$ is a dimensionless coefficient that depends on the tank proportions (ratios of liquid height to tank radius) and on the number, relative thicknesses and relative mass densities of the liquid layers. The values of $C_{m n}$ and the associated vectors $\left\{\hat{D}_{m n}\right\}$ have been presented in Reference 1 for a number of two-layered and three-layered systems. It should be recalled that while they increase with increasing $m$, the values of $C_{m n}$ decrease with increasing $n$.

It has further been shown [1] that

$$
\sum_{n=1}^{N}\left\{d_{m n}\right\}=\epsilon_{m}\{1\}
$$

and that

$$
\sum_{m=1}^{\infty} \epsilon_{m}=1
$$




\subsection{Hydrodynamic Pressures}

On substituting Eq. (3) into Eq. (2) and making use of Eq. (1), the hydrodynamic pressure in the $j$ th layer, $p_{j}\left(\xi, \eta_{j}, \theta, t\right)$, may be expressed as

$p_{j}=-\left\{\xi \ddot{x}_{g}(t)+\sum_{m=1}^{\infty}\left[\frac{\ddot{D}_{m, j}(t) \cosh \lambda_{m} \eta_{j}-\ddot{D}_{m, j-1}(t) \cosh \lambda_{m}\left(\alpha_{j}-\eta_{j}\right)}{\lambda_{m} \sinh \lambda_{m} \alpha_{j}}\right] \frac{J_{1}\left(\lambda_{m} \xi\right)}{J_{1}\left(\lambda_{m}\right)}\right\} \rho_{j} R \cos \theta$

in which $\ddot{D}_{m, j}$ and $\ddot{D}_{m, j-1}$ are obtained from Eq. (7) by double differentiation with respect to time. On recalling that $A_{m n}(t)$ is related to the deformation $U_{m n}(t)$ of a base-excited single-degree-of-freedom system by

$$
A_{m n}(t)=-\omega_{m n}^{2} U_{m n}(t)
$$

and that the second time derivative of $U_{m n}(t)$ may be determined from the equation of motion for such a system to be

$$
\ddot{U}_{m n}(t)=-\ddot{x}_{g}(t)-\omega_{m n}^{2} U_{m n}(t)=-\ddot{x}_{g}(t)+A_{m n}(t)
$$

Eq. (7) leads to

$$
\left\{\ddot{D}_{m}(t)\right\}=-R \sum_{n=1}^{N} \omega_{m n}^{2}\left\{d_{m n}\right\}\left(\frac{\ddot{x}_{g}(t)}{g}-\frac{A_{m n}(t)}{g}\right)
$$

Finally, on substituting the expressions for $\ddot{D}_{m, j}(t)$ and $\ddot{D}_{m, j-1}(t)$ into Eq. (13), making use of Eq. (10), and grouping together all terms that are proportional to the ground acceleration, Eq. (13) may be rewritten as the sum of an impulsive component and a convective component as

$$
p_{j}\left(\xi, \eta_{j}, \theta, t\right)=p_{j}^{i}\left(\xi, \eta_{j}, \theta, t\right)+p_{j}^{c}\left(\xi, \eta_{j}, \theta, t\right)
$$

The impulsive component, which represents the effect of the part of the liquid that may be considered to move as a rigid body with the tank wall and hence experiences the same acceleration as the ground, is given by

$$
p_{j}^{i}\left(\xi, \eta_{j}, \theta, t\right)=-\left[\xi-\sum_{m=1}^{\infty} \sum_{n=1}^{N} c_{m n, j}\left(\eta_{j}\right) \frac{J_{1}\left(\lambda_{m} \xi\right)}{J_{1}\left(\lambda_{m}\right)}\right] \rho_{j} R \cos \theta \ddot{x}_{g}(t)
$$

whereas the convective component, which represents the effect of the sloshing action of the liquid, is given by

$$
p_{j}^{c}\left(\xi, \eta_{j}, \theta, t\right)=-\left[\sum_{m=1}^{\infty} \sum_{n=1}^{N} c_{m n, j}\left(\eta_{j}\right) \frac{J_{1}\left(\lambda_{m} \xi\right)}{J_{1}\left(\lambda_{m}\right)} A_{m n}(t)\right] \rho_{j} R \cos \theta
$$


in which

$$
c_{m n, j}\left(\eta_{j}\right)=C_{m n}^{2}\left[\frac{d_{m n, j} \cosh \lambda_{m} \eta_{j}-d_{m n, j-1} \cosh \lambda_{m}\left(\alpha_{j}-\eta_{j}\right)}{\sinh \lambda_{m} \alpha_{j}}\right]
$$

In the latter expression, $d_{m n, j}$ represents the $j$ th element of $\left\{d_{m n}\right\} ; C_{m n}$ represents the dimensionless frequency coefficient in Eq. (10); and the terms with the factors $d_{m n, j-1}$ and $d_{m n, j}$ represent the effect of the sloshing or rocking actions of the lower and upper interfaces, respectively. It should be clear from Eq. (19) that there is an infinite number of horizontal sloshing modes of vibration; that for each such mode, there exist $N$ vertical modes; and that associated with each horizontal and vertical mode, there is a distinct pseudoacceleration function, $A_{m n}(t)$. The summations on $m$ in Eqs. (18) and (19) represent the contributions of the horizontal modes, while those on $n$ represent the contributions of the vertical modes.

3.2.1 Simplification of solution for impulsive pressures. In the form presented so far, the evaluation of the impulsive component of response requires the prior evaluation of the convective components. The impulsive component can also be evaluated independently of the convective as follows : On letting

$$
\left\{e_{m}\right\}=\sum_{n=1}^{N} C_{m n}^{2}\left\{d_{m n}\right\}
$$

Eq. (18) may be rewritten as a single series as

$p_{j}^{i}\left(\xi, \eta_{j}, \theta, t\right)=-\left\{\xi-\sum_{m=1}^{\infty}\left[e_{m, j} \frac{\cosh \lambda_{m} \eta_{j}}{\sinh \lambda_{m} \alpha_{j}}-e_{m, j-1} \frac{\cosh \lambda_{m}\left(\alpha_{j}-\eta_{j}\right)}{\sinh \lambda_{m} \alpha_{j}}\right] \frac{J_{1}\left(\lambda_{m} \xi\right)}{J_{1}\left(\lambda_{m}\right)}\right\} \rho_{j} R \cos \theta \ddot{x}_{g}(t)$

in which $e_{m, j}$ is the $j$ th element of $\left\{e_{m}\right\}$. It is shown in Appendix I that the vector $\left\{e_{m}\right\}$ also represents the solution of the system of algebraic equations

$$
[\mathcal{A}]\left\{e_{m}\right\}=\epsilon_{m}\{s\}
$$

in which $[\mathcal{A}]$ and $\{s\}$ are the matrices appearing in Eq. (5). With the values of $\left\{e_{m}\right\}$ determined in this manner, the impulsive pressures may be evaluated from Eq. (22) without prior knowledge of the sloshing frequencies and the associated modes of vibration of the system. The sumerical solutions reported herein have been obtained by this approach.

It may be of interest to observe that Eq. (23) is merely a statement of the fact that the impulsive pressures are continuous at the $N$ liquid interfaces. If the dimensionless distance coordinate $\xi$ in Eq. (22) is expanded in the form

$$
\xi=\sum_{m=1}^{\infty} \epsilon_{m} \frac{J_{1}\left(\lambda_{m} \xi\right)}{J_{1}\left(\lambda_{m}\right)}
$$


the $j$ th component of Eq. (23) is obtained simply by equating the expressions for the impulsive component of the pressure on either side of the $j$ th interface.

3.2.2 Wall pressures. The hydrodynamic wall pressures for the $j$ th layer may be written in the form

$$
p_{j}\left(1, \eta_{j}, \theta, t\right)=-\left[c_{o, j}\left(\eta_{j}\right) \ddot{x}_{g}(t)+\sum_{m=1}^{\infty} \sum_{n=1}^{N} c_{m n, j}\left(\eta_{j}\right) A_{m n}(t)\right] \rho_{j} R \cos \theta
$$

where $c_{o, j}\left(\eta_{j}\right)$ is a dimensionless function, obtained from the expression within the braces in Eq. (22) by letting $\xi=1$, i.e.,

$$
c_{o, j}\left(\eta_{j}\right)=1-\sum_{m=1}^{\infty}\left[e_{m, j} \frac{\cosh \lambda_{m} \eta_{j}}{\sinh \lambda_{m} \alpha_{j}}-e_{m, j-1} \frac{\cosh \lambda_{m}\left(\alpha_{j}-\eta_{j}\right)}{\sinh \lambda_{m} \alpha_{j}}\right]
$$

From Eqs. (20), (21) and (26), it now follows that

$$
c_{o, j}\left(\eta_{j}\right)+\sum_{m=1}^{\infty} \sum_{n=1}^{N} c_{m n, j}\left(\eta_{j}\right)=1
$$

It is important to note that the pressure in Eq. (25) is expressed in terms of the density of the layer under consideration rather than that of some reference layer.

3.2.3 Specialized expressions for wall pressures. For a single-layered, homogeneous system, $[\mathcal{A}]=\operatorname{coth} \lambda_{m} H / R,\{s\}=1$, Eq. (23) yields

$$
e_{m}=\epsilon_{m} \tanh \lambda_{m} H / R
$$

and Eq. (26) reduces, as it should, to the well-known expression (see, for example, Reference 9),

$$
c_{o}(z)=1-\sum_{m=1}^{\infty} \epsilon_{m} \frac{\cosh \lambda_{m} z / R}{\cosh \lambda_{m} H / R}
$$

where $z$ is the vertical distance from the tank base, and the $m$ th term of the summation represents the dimensionless function $c_{m}(z)$ in the expression for the convective component of the pressure.

For a two-layered system, the solution of Eq. (23) yields

$$
e_{m, 1}=\epsilon_{m} \frac{\left[\left(1-\rho_{2} / \rho_{1}\right) \cosh \lambda_{m} \alpha_{2}+\rho_{2} / \rho_{1}\right] \sinh \lambda_{m} \alpha_{1}}{\cosh \lambda_{m} \alpha_{1} \cosh \lambda_{m} \alpha_{2}+\left(\rho_{2} / \rho_{1}\right) \sinh \lambda_{m} \alpha_{1} \sinh \lambda_{m} \alpha_{2}}
$$

and

$$
e_{m, 2}=\frac{\epsilon_{m} \sinh \lambda_{m} \alpha_{2}+e_{m, 1}}{\cosh \lambda_{m} \alpha_{2}}
$$


On substituting these expressions for $e_{m, j}$ into Eq. (26) and recalling that $e_{m, j-1}$ for $j=1$ is zero, the resulting expressions can be shown to reduce to those presented in References 2 and 3. The corresponding expressions for the convective pressure components are given by Eq. (20), in which $d_{m n, j-1}$ for $j=1$ must be taken as zero, $d_{m n, j}$ for $j=1$ and 2 must be determined from Eq. (8) and $C_{m n}^{2}$ must be evaluated from Eq. (40) of Reference 1. The results obtained in this manner can again be shown to agree with those obtained from expressions presented in Reference 4.

\subsection{Tank Forces}

3.3.1 Base shear. The instantaneous value of the base shear or total hydrodynamic force acting on the tank-wall, $Q_{b}(t)$, is given by

$$
Q_{b}(t)=\sum_{j=1}^{N} \int_{0}^{H,} \int_{0}^{2 \pi} p_{j}\left(1, z_{j}, \theta, t\right) R \cos \theta d \theta d z_{j}
$$

which, on expressing the wall pressure by Eq. (25) and performing the indicated integrations, can be written as

$$
Q_{b}(t)=m_{o} \ddot{x}_{g}(t)+\sum_{m=1}^{\infty} \sum_{n=1}^{N} m_{m n} A_{m n}(t)
$$

with $m_{o}$ and $m_{m n}$ given by

$$
m_{o}=\sum_{j=1}^{N} m_{o, j}=\sum_{j=1}^{N} m_{l, j}\left(1-\sum_{m=1}^{\infty} \frac{e_{m, j}-e_{m, j-1}}{\lambda_{m} \alpha_{j}}\right)
$$

and

$$
m_{m n}=\sum_{j=1}^{N} m_{m n, j}=\sum_{j=1}^{N} m_{l, j}\left(\frac{C_{m n}^{2} d_{m n, j}-C_{m n}^{2} d_{m n, j-1}}{\lambda_{m} \alpha_{j}}\right)
$$

The quantity $m_{l, j}$ in the preceding two equations represents the mass of the $j$ th layer, $\rho_{j} \pi R^{2} H_{j} ; m_{o, j}$ represents the portion of $m_{l, j}$ that acts impulsively; and $m_{o}$ represents the total impulsive mass. Similarly, $m_{m n}$ represents the total liquid mass participating in the $m$ th horizontal and $n$th vertical sloshing mode of vibration, and $m_{m n, j}$ represents the part that is contributed by the $j$ th layer. From Eqs. (34) and (35), and with the aid of Eq. (21), it can finally be shown that

$$
m_{o}+\sum_{m=1}^{\infty} \sum_{n=1}^{N} m_{m n}=\sum_{j=1}^{N} m_{l, j}=m_{l}
$$

That is, the sum of the impulsive mass and all convective masses equals the total mass of the liquid, $m_{l}$. 
3.3.2 Moment above base. The instantaneous value of the hydrodynamic moment induced across a section of the tank immediately above the base is given by

$$
M(t)=\sum_{j=1}^{N} \int_{0}^{H_{j}} \int_{0}^{2 \pi} p_{j}\left(1, z_{j}, \theta, t\right)\left[L_{j-1}+z_{j}\right] R \cos \theta d \theta d z_{j}
$$

where

$$
L_{j-1}=\sum_{k=1}^{j-1} H_{k}
$$

refers to the height of the $(j-1)$ th liquid interface measured from the tank base. On substituting Eq. (25) for the wall pressure into Eq. (37) and integrating, one obtains

$$
M(t)=m_{o} h_{o} \ddot{x}_{g}(t)+\sum_{m=1}^{\infty} \sum_{n=1}^{N} m_{m n} h_{m n} A_{m n}(t)
$$

in which the quantity $m_{o} h_{o}$ for the impulsive component of response is given by

$$
m_{o} h_{o}=\sum_{j=1}^{N}\left\{m_{l, j} H_{j}\left[\frac{1}{2}-\sum_{m=1}^{\infty}\left(\frac{\left(e_{m, j}+e_{m, j-1}\right)\left(1-\cosh \lambda_{m} \alpha_{j}\right)}{\lambda_{m}^{2} \alpha_{j}^{2} \sinh \lambda_{m} \alpha_{j}}+\frac{e_{m, j}}{\lambda_{m} \alpha_{j}}\right)\right]+m_{o, j} L_{j-1}\right\}
$$

and the quantity $m_{m n} h_{m n}$ for the convective component associated with the $m$ th horizontal and $n$th vertical mode of vibration is given by

$m_{m n} h_{m n}=\sum_{j=1}^{N}\left\{m_{l, j} H_{j}\left[C_{m n}^{2}\left(\frac{\left(d_{m n, j}+d_{m n, j-1}\right)\left(1-\cosh \lambda_{m} \alpha_{j}\right)}{\lambda_{m}^{2} \alpha_{j}^{2} \sinh \lambda_{m} \alpha_{j}}+\frac{d_{m n, j}}{\lambda_{m} \alpha_{j}}\right)\right]+m_{m n, j} L_{j-1}\right\}$

The quantity $h_{o}$ in these expressions represents the height at which the mass $m_{o}$ must be concentrated to yield the impulsive component of the base moment, and $h_{m n}$ represents the height at which $m_{m n}$ must be concentrated to yield the convective component of the corresponding moment associated with the $m$ th horizontal and $n$th vertical mode of vibration. From Eqs. (40) and (41)and with the aid of Eqs. (21) and (36), it can be shown that

$$
m_{o} h_{o}+\sum_{m=1}^{\infty} \sum_{n=1}^{N} m_{m n} h_{m n}=\sum_{j=1}^{N} m_{l, j}\left(L_{j-1}+\frac{H_{j}}{2}\right)=m_{l} h_{l}
$$

where $h_{l}$ represents the height of the center of gravity of the total liquid mass from the tank base.

3.3.3 Foundation moment. In addition to the moment $M(t)$, the foundation moment, $M^{\prime}(t)$, includes the effect of the hydrodynamic pressures exerted on the tank base. The latter moment is given by,

$$
M^{\prime}(t)=M(t)+\int_{0}^{R} \int_{0}^{2 \pi} p_{1}(r, 0, \theta, t) r^{2} \cos \theta d \theta d r
$$


which, on expressing $M(t)$ by Eq. (39), replacing $p_{1}$ by the sum of Eqs. (18) and (19) with $j=1$, and integrating, can be written as

$$
M^{\prime}(t)=m_{o} h_{o}^{\prime} \ddot{x}_{g}(t)+\sum_{m=1}^{\infty} \sum_{n=1}^{N} m_{m n} h_{m n}^{\prime} A_{m n}(t)
$$

with

$$
m_{o} h_{o}^{\prime}=m_{o} h_{o}+m_{l, 1} H_{1}\left(\frac{1}{4 \alpha_{1}^{2}}-\sum_{m=1}^{\infty} \frac{e_{m, 1}}{\alpha_{1}^{2} \lambda_{m}^{2} \sinh \lambda_{m} \alpha_{1}}\right)
$$

and

$$
m_{m n} h_{m n}^{\prime}=m_{m n} h_{m n}+m_{l, 1} H_{1}\left(\frac{C_{m n}^{2} d_{m n, 1}}{\alpha_{1}^{2} \lambda_{m}^{2} \sinh \lambda_{m} \alpha_{1}}\right)
$$

From the latter two expressions and Eqs. (21) and (42), one finally obtains

$$
m_{o} h_{o}^{\prime}+\sum_{m=1}^{\infty} \sum_{n=1}^{N} m_{m n} h_{m n}^{\prime}=m_{l} h_{l}+m_{l, 1} H_{1} \frac{1}{4 \alpha_{1}^{2}}=m_{l} h_{l}^{\prime}
$$

where the term on the extreme right represents the foundation moment induced by an unit acceleration when the entire liquid is presumed to act as a rigid mass, and the term involving $m_{l, 1}$ represents the component of this moment contributed by the base pressure. The latter pressure increases linearly from zero at the center of the tank base to $\rho_{1} R \cos \theta$ at the junction of the base and the wall. 


\section{SECTION 4}

\section{PRESENTATION AND ANALYSIS OF NUMERICAL SOLUTIONS}

\subsection{Hydrodynamic Wall Pressures}

4.1.1 Normalization of Wall Pressures. In examining their variations with height, it is desirable to express the hydrodynamic wall pressures in terms of the density of some reference liquid layer rather than in the form of Eq. (25), in terms of the density of the layer being considered. In the remainder of this paper, all pressures are expressed in terms of the mass density of the heaviest or bottom layer, $\rho_{1}$, as

$$
p(1, \eta, \theta, t)=-\left[c_{o}(\eta) \ddot{x}_{g}(t)+\sum_{m=1}^{\infty} \sum_{n=1}^{N} c_{m n}(\eta) A_{m n}(t)\right] \rho_{1} R \cos \theta
$$

where $\eta=z / H$ is the normalized vertical position coordinate, and $c_{o}(\eta)$ and $c_{m n}(\eta)$ are dimensionless functions defining the vertical distributions of the various pressure components.

For a value of $z$ corresponding to the $j$ th layer (i.e., $L_{j-1} \leq z \leq L_{j}$ ), the functions $c_{o}(\eta)$ and $c_{m n}(\eta)$ are related to the functions $c_{o, j}\left(\eta_{j}\right)$ and $c_{m n, j}\left(\eta_{j}\right)$ in Eq. (25) by

$$
c_{o}(\eta)=\frac{\rho_{j}}{\rho_{1}} c_{o, j}\left(\eta_{j}\right) \quad \text { and } \quad c_{m n}(\eta)=\frac{\rho_{j}}{\rho_{1}} c_{m n, j}\left(\eta_{j}\right)
$$

Accordingly, Eq. (27) may be rewritten as

$$
c_{o}(\eta)+\sum_{m=1}^{\infty} \sum_{n=1}^{N} c_{m n}(\eta)=\frac{\rho_{j}}{\rho_{1}} \quad \text { for } L_{j-1} \leq z \leq L_{j}
$$

It is shown in Appendix II that the $c_{m n}(\eta)$ functions are discontinuous at the layer interfaces, and that, for each horizontal mode of vibration, the sum of the discontinuities at an interface for all the vertical modes of vibration satisfy the relation

$$
\sum_{n=1}^{N}\left\{c_{m n}^{-}-c_{m n}^{+}\right\}=\epsilon_{m}\{s\}
$$

in which the - and + superscripts identify sections immediately below and above the interface under consideration. For two-layered systems, Eq. (51) reduces to

$$
\sum_{n=1}^{2}\left(c_{m n}^{1-}-c_{m n}^{1+}\right)=\epsilon_{m}\left(1-\frac{\rho_{2}}{\rho_{1}}\right)
$$


for the first or lower interface, and to

$$
\sum_{n=1}^{2} c_{m n}^{2-}=\epsilon_{m}\left(\frac{\rho_{2}}{\rho_{1}}\right)
$$

for the second or top interface.

4.1.2 Representative Wall Pressures. Fig. 4.1 shows the heightwise distributions of the components of wall pressure for a tank with $H / R=1$ containing a two-layered liquid with $\rho_{2} / \rho_{1}=0.5$ and $H_{2}=H_{1}=0.5 \mathrm{H}$. Part (a) of the figure shows the dimensionless function $c_{o}(\eta)$ for the impulsive component of the pressure, whereas part (b) shows the functions $c_{m n}(\eta)$ for the convective components associated with the first two horizontal modes of vibration. It should be recalled that there is an infinite number of horizontal modes, and that to each such mode there correspond $N$ (two for a two-layered system) vertical modes. The $c_{m n}(\eta)$ functions for the third and higher horizontal modes are negligibly small and are not included. Also shown in part (c) of the figure is the distribution function $c_{l}(\eta)$ computed on the assumption that the entire liquid mass acts impulsively.

Similar plots are given in Fig. 4.2 for a three-layered liquid with equal layer thicknesses and values of $\rho_{j}$ increasing from top to bottom in the ratio $1 / 2 / 3$. In this case, only the convective pressure distributions corresponding to the fundamental horizontal mode of vibration are given.

The following trends are worth noting in Figs. 4.1 and 4.2 :

1. As is true of a homogeneous liquid, the impulsive pressures increase from zero at the top to a maximum at the base. The distributions of these pressures are continuous, but exhibit slope discontinuities or cusps at the layer interfaces.

2. The convective pressure components are discontinuous at the layer interfaces and, for a given horizontal mode of vibration, the sum of the discontinuities at an interface for all the vertical modes satisfies $\mathrm{Eq}$. (51).

3. Irrespective of the order of the horizontal mode of vibration, the convective pressure associated with the $n$th vertical mode exhibits $(n-1)$ changes in sign. These changes are consistent with those noted in Reference 1 for the corresponding modal displacements, and are associated vith the relative sloshing or rocking actions of successive interfaces.

4. The algebraic sum of the impulsive and of all the convective pressure distribution functions satisfies Eq. (50); it is, therefore, equal to the function obtained by considering the entire liquid to act as a rigid mass. 
In assessing the relative importance of the various convective pressure components, it should be kept in mind that their contributions depend not only on the values of the dimensionless distribution functions $c_{m n}(\eta)$ but also on those of the corresponding pseudoacceleration functions $A_{m n}(t)$. The latter functions depend, in turn, on the characteristics of the ground motion, and on the natural frequency and damping of the mode of vibration being considered.

As an illustration, consider the two-layered system examined previously in Reference 1 , for which $H=36 \mathrm{ft}(10.98 \mathrm{~m}), R=60 \mathrm{ft}(18.29 \mathrm{~m}), H_{2}=2 H_{1}=2 H / 3$ and $\rho_{2}$ $=0.5 \rho_{1}$. The instantaneous value of the normalized hydrodynamic wall pressure at a section just below the interface of the two layers in this case is given by

$$
\frac{p\left(1, \frac{1}{3}^{-}, \theta, t\right)}{\gamma_{1} R \cos \theta}=0.265 \frac{\ddot{x}_{g}(t)}{g}+0.431 \frac{A_{11}(t)}{g}+0.239 \frac{A_{12}(t)}{g}+0.009 \frac{A_{21}(t)}{g}+0.023 \frac{A_{22}(t)}{g}+\ldots
$$

in which $\gamma_{1}=\rho_{1} g$ is the unit weight of the lower layer. Further, let the ground motion be specified by the design response spectrum presented in Fig. 8 of Reference 1 , which corresponds to a maximum ground acceleration $\ddot{x}_{g}=0.33 g$ and a coefficient of viscous damping of 0.5 percent critical. Using the natural sloshing frequency values listed in Table III of the same reference, the maximum or spectral values of the first four pseudoacceleration functions $A_{m n}(t)$, denoted by $A_{m n}$, are found to be

$$
A_{11}=0.059 g \quad A_{12}=0.005 g \quad A_{21}=0.228 g \quad A_{22}=0.064 g
$$

On substituting these values along with $\ddot{x}_{g}=0.33 g$ into Eq. (54), the maximum values of the impulsive and the first four convective terms become

\begin{tabular}{c|c|c|c|c}
\hline \multirow{3}{*}{$\begin{array}{c}\text { Impulsive } \\
\text { Term }\end{array}$} & \multicolumn{3}{|c}{ Convective Terms } \\
\cline { 2 - 5 } & \multicolumn{2}{|c|}{$\mathrm{m}=1$} & \multicolumn{2}{c}{$\mathrm{m}=2$} \\
\cline { 2 - 5 } & $\mathrm{n}=1$ & $\mathrm{n}=2$ & $\mathrm{n}=1$ & $\mathrm{n}=2$ \\
\hline 0.0875 & 0.0259 & 0.0012 & 0.0021 & 0.0015 \\
\hline
\end{tabular}

Finally, when coinputed approximately by adding to the maximum value of the impulsive component the square root of the sum of squares of the convective components, the maximum value of the total hydrodynamic wall pressure at the elevation consi $t$. ered becomes $0.114 \gamma_{1} R$.

It should be noted that, whereas the coefficient of the term involving the $A_{12}(t)$ function is much larger than of the term involving the $A_{21}(t)$ function, the opposite is true of the relative contributions of these two terms to the wall pressure. Note 
further that the maximum component of the convective pressure is contributed by the fundamental sloshing mode of vibration $(m=n=1)$, that the contributions of the higher modes are negligibly small, and that the total convective pressure is small compared to the corresponding impulsive pressure. These results are representative of those that can be expected for large capacity tanks of normal proportions subjected to earthquakes.

4.1.3 Wall Pressures for Two-Layered Systems. In the left part of Fig. 4.3, the $c_{o}(\eta)$ function for the impulsive component of the wall pressure for the two-layered system examined previously in Fig. 4.1 is compared with those obtained for several other values of the density ratio $\rho_{2} / \rho_{1}$. Also shown are the corresponding functions $c_{11}(\eta)$ and $c_{12}(\eta)$ for the first horizontal sloshing mode of vibration. As would be expected, the impulsive pressure coefficients decrease with decreasing $\rho_{2} / \rho_{1}$, and for the limiting case of $\rho_{2} / \rho_{1}=0$, they reduce to the values applicable to a tank that is half-full with a homogeneous liquid of density $\rho_{1}$. By contrast, the convective pressures in the lower layer increase with decreasing $\rho_{2} / \rho_{1}$, and as $\rho_{2} / \rho_{1}$ tends to zero, $c_{11}(\eta)$ and $c_{12}(\eta)$ become proportional to each other and their sum tends to the corresponding function for the half-full tank. The latter function is associated with a value of 0.837 at the tank mid-height and a value of 0.575 at the tank base.

The limiting behavior of the convective pressure distributions referred to above is strictly valid only for systems with $H_{1}=H_{2}=0.5 \mathrm{H}$, for which the uncoupled natural frequencies of the two layers (i.e., the frequencies computed considering the two layers to act independently) are equal. For systems with unequal layer thicknesses, as $\rho_{2} / \rho_{1}$ tends to zero, the convective pressure distribution for the tank containing only the lower layer is reached by the function $c_{1 n}(z)$ for which the associated frequency of vibration is closest to the uncoupled natural frequency of the lower layer. This is demonstrated in Fig. 4.4, where the distributions of $c_{11}(\eta)$ and $c_{12}(\eta)$ for a tank with $H / R=1$ and $\rho_{2} / \rho_{1}=0.1$ are shown for two values of $H_{2} / H_{1}$. Note that for $H_{2}=0.5 H_{1}$, for which the fundamental uncoupled natural frequency of the lower layer is higher than that of the upper (see, for example, Eq. 44 in Reference 1), it is the $c_{11}(\eta)$ function that approaches the distribution of the partially filled tank, while $c_{12}(\eta)$ becomes negligibly small. By contrast, for $H_{2}=2 H_{1}$, for which the uncoupled natural frequency of the bottom layer is the lower of the two, it is the $c_{12}(\eta)$ function that approaches the distribution of the partially filled tank while $c_{11}(\eta)$ tends to zero.

The impulsive and convective pressure coefficients for additional two-layered systems are listed in Table 4.1. The tabulated results are for the free surface, for sections 
immediately above and below the interface (denoted by $I+$ and $I$-, respectively), and for the tank base of systems with $H / R=0.5,1$ and 2 , and $H_{2} / H_{1}=0.5$ and 2 . The general trends of these data are similar to those of the data displayed in Figs. 4.3 and 4.4 .

\subsection{Tank Forces}

4.2.1 Two-layered systems. Fig. 4.5 shows the masses $m_{0}$ and $m_{11}$ in the expression for base shear of systems with equal layer thicknesses and density ratios $\rho_{2} / \rho_{1}$ in the range between 0.1 and 1 . The results are plotted as a function of the total liquid height to tank radius ratio, $H / R$, and they are normalized with respect to $m_{l}$, the total liquid mass of the system being considered. Normalized values of the corresponding base moment coefficients, $m_{o} h_{o}$ and $m_{11} h_{11}$, and of the foundation moment coefficients, $m_{\circ} h_{\circ}^{\prime}$ and $m_{11} h_{11}^{\prime}$, are presented in Figs. 4.6 and 4.7, respectively. The normalizing quantities in these plots are those obta 1 by considering the entire liquid to act as a rigid mass, and are naturally different for tanks of different proportions and contents.

The normalized values of $m_{0}$ and $m_{11}$ for additional two-layered systems, along with the corresponding values of $m_{12}, m_{21}$ and $m_{22}$, are presented in Table 4.2 , and the associated moment coefficients are presented in Tables 4.3 and 4.4. Examination of these data and of those displayed in the figures reveals the following trends :

1. For values of $\rho_{2} / \rho_{1}$ between 0.5 and 1 , the normalized value of the liquid masses $m_{o}, m_{11}$ and $m_{21}$ may, for all practical purposes, be considered to be same over the entire range of $H / R$ considered. The same is also true of the crirresponding moment cnefficients, although the ranges of $\rho_{2} / \rho_{1}$ and $H / R$ over which the results may be considered to be the same are somewhat different in the two cases. Incidentally, these quantities are the ones most likely to affect significantly the seismic response of practical systems. It follows that, within the indicated range of $\rho_{2} / \rho_{1}$ values, the solutions for layered systems may be obtisined with reasonable accuracy from well-established solutions [9] for tanks with homogeneous liquids. It should be recalled, however, that the normalizing quantities are different in the two cases.

2. For values of $\rho_{2} / \rho_{1}$ smaller than 0.5 , the proportion of the total liquid acting impulsively may be substantially lower for the layered system than for the homogeneous system. The large interfacial discontinuity in liquid density increases the sloshing or convective actions of the system, and this increase, in turn, leads to a corresponding diminution of the impulsive effects. 
3. While the increase in the convective action of systems with the large changes in liquid density does not necessarily increase the normalized values of the responses components for $n=1$, it does increase the sum of the corresponding components for $n=1$ and $n=2$. This is true for each horizontal mode of vibration and is demonstrated in Fig. 4.8 for the convective masses associated with the first and second horizontal modes of vibration (i.e., $m=1$ and $m=2$ ). It can be seen that, in each case, the sum of the convective masses for the layered system is indeed higher than the corresponding mass for the homogeneous system.

4.2.2 Three-layered systems. Numerical data similar to those presented in the preceding section for two-layered systems are given in Tables $4.5,4.6$ and 4.7 for three-layered systems with equal layer thicknesses. Three different values of $H / R$ and two different ratios of layer densities are considered. As before, the results are no'malized with respect to those computed on the assumption that the entire liquid mass acts rigidly.

The tabulated data satisfy Eqs. (36), (42) and (47), and the interrelationships of the impulsive and convective results are generally similar to those for the two-layered systems examined in previous sections.

\subsection{Solutions for Continuous Systems}

One of the great merits of the analysis for multi-layered systems presented herein is its ability to closely approximate the response of systems with continuous variations in liquid density. This is demonstrated in this section for a system with $H / R=1$ for which the density variation is defined by

$$
\rho(\eta)=\rho_{\circ} e^{-\beta \eta}
$$

In this expression, $\eta=z / H$ is the dimensionless distance coordinate, measured upward from the base; and $\beta$ is a dimensionless, positive decay factor. For the solutions presented herein, $\beta$ is taken as 1.386 so that $\rho(1) / \rho_{o}$ is 0.25 . The wall pressure for the continuous system is defined by Eq. (48), in which $N$ must now be replaced by infinity and $\rho_{1}$ must be interpreted as the base value of the liquid density, $\rho_{o}$.

For the discretized solutions, the liquid is approximated by $N$ uniform layers of equal thicknesses and density values equal to those determined from the continuous distribution at mid-heights of the substitute layers. Fig. 4.9 shows the heightwise variations of the impulsive component of wall pressure and of the convective components associated with the fundamental horizontal and first three vertical modes of vibration. 
The dashed lines represent the exact solutions for the continuous density variation, whereas the solid lines represent the solutions for the approximating layered systems with $N=10$ and $N=50$. The derivation of the exact solutions for the continuous system will be presented in a subsequent paper. The results for both the layered and continuous systems are expressed in terms of the base value of the liquid density, $\rho_{o}$. It is seen that the impulsive pressures for the layered system with $N=10$ are practically indistinguishable from those of the continuous system. By contrast, the convective pressures of the layered system converge less rapidly, and a much larger number of layers is required to achieve comparable accuracy.

Table 4.8 gives the normalized values of the impulsive and of the first six convective masses computed for layered systems with values of $N$ ranging from 5 to 50 . Also listed are the corresponding exact solutions for the continuous variation in density. Tables 4.9 and 4.10 give the corresponding moment coefficients for sections above and below the tank base, respectively. It can be seen that the solutions for the discrete systems do converge to those of the continuous system; that the rates of convergence of the results are quite rapid; and that good agreement is obtained with as few as ten uniform layers.

For the evaluation of the pseudoacceleration functions in the expressions for the convective components of response, one needs to know the natural frequencies of sloshing motion and the associated modes of vibration. The convergence of these quantities for the system examined herein was studied in Reference 8, and it is not reconsidered. It is worth noting, however, that both the natural frequencies and the modes of vibration of the discrete systems converge to those of the continuous system more rapidly than do the corresponding convective pressures. Two factors are responsible for the improved convergence : (1) Unlike the convective wall pressures that are discontinuous at the interfaces, the modal displacements are continuous; and (2) the natural frequencies are relatively insensitive to inaccuracies in the corresponding modes of vibration. 


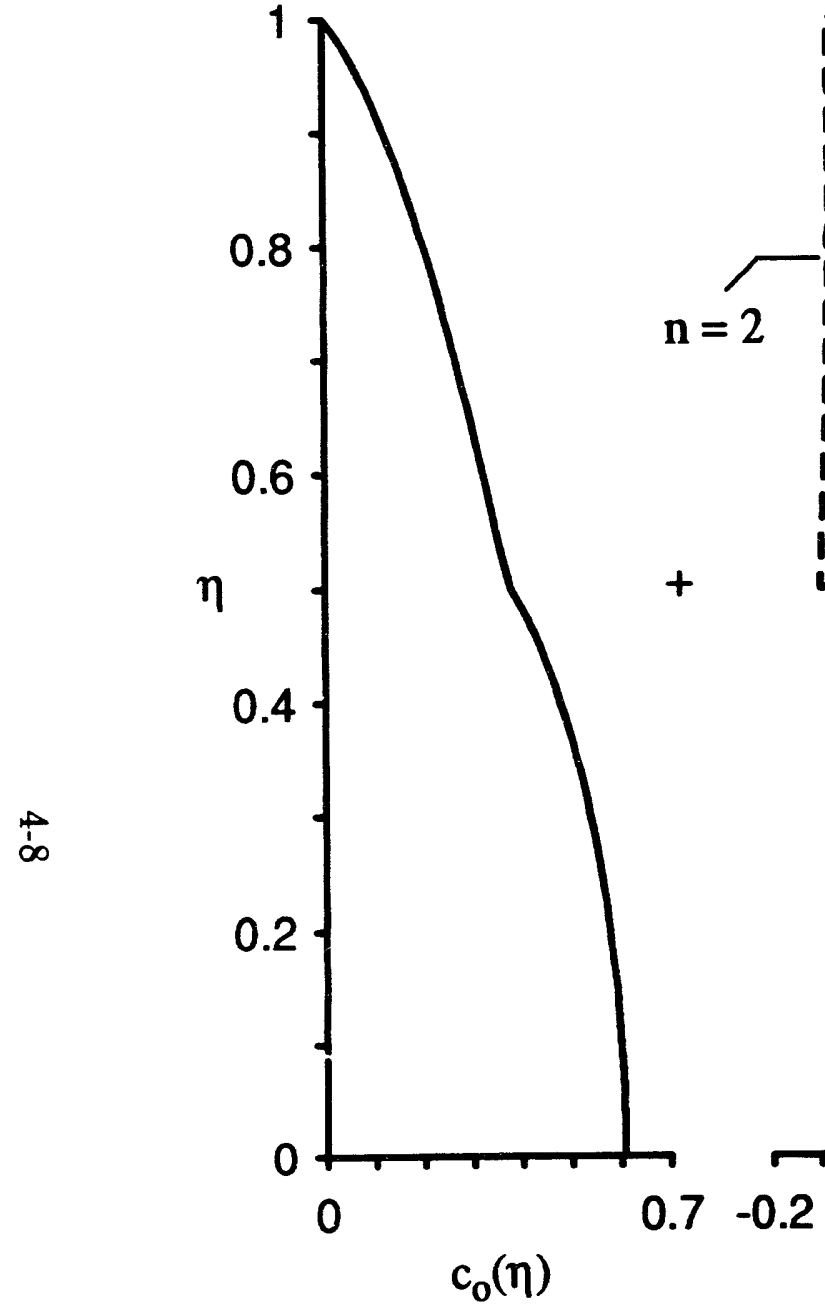

(a) Impulsive

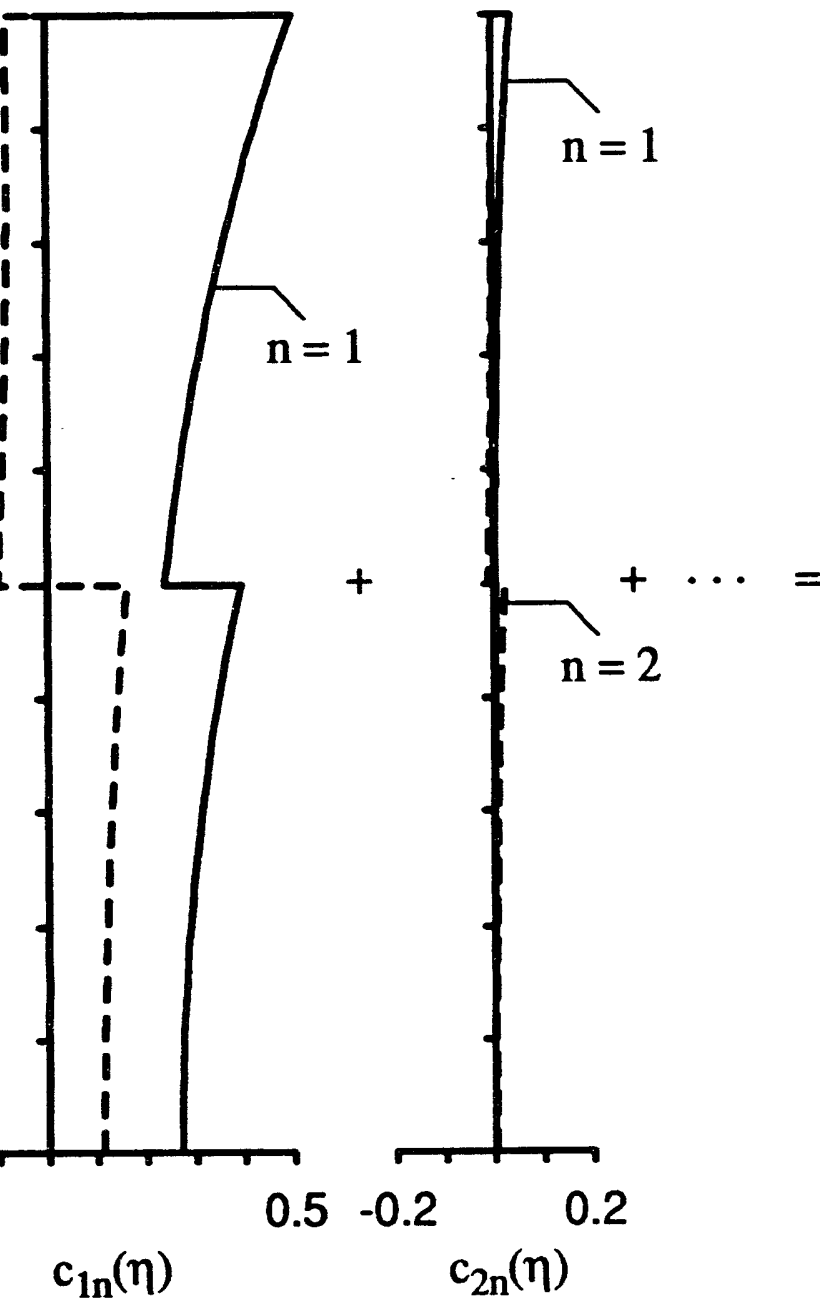

(b) Convective

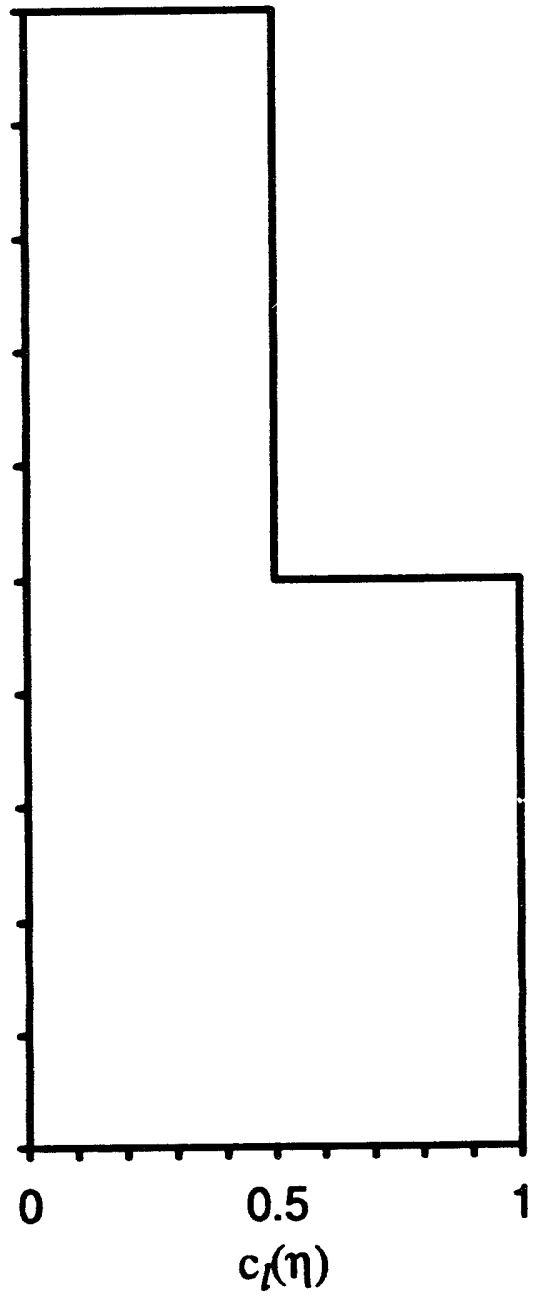

(c) Total

Figure 4.1 Interrelationship of coefficients for impulsive and convective components of wall pressure for two-layered system with $\mathrm{H} / \mathrm{R}=1, \mathrm{H}_{2} / \mathrm{H}_{1}=1$ and $\rho_{2} / \rho_{1}=0.5$ 


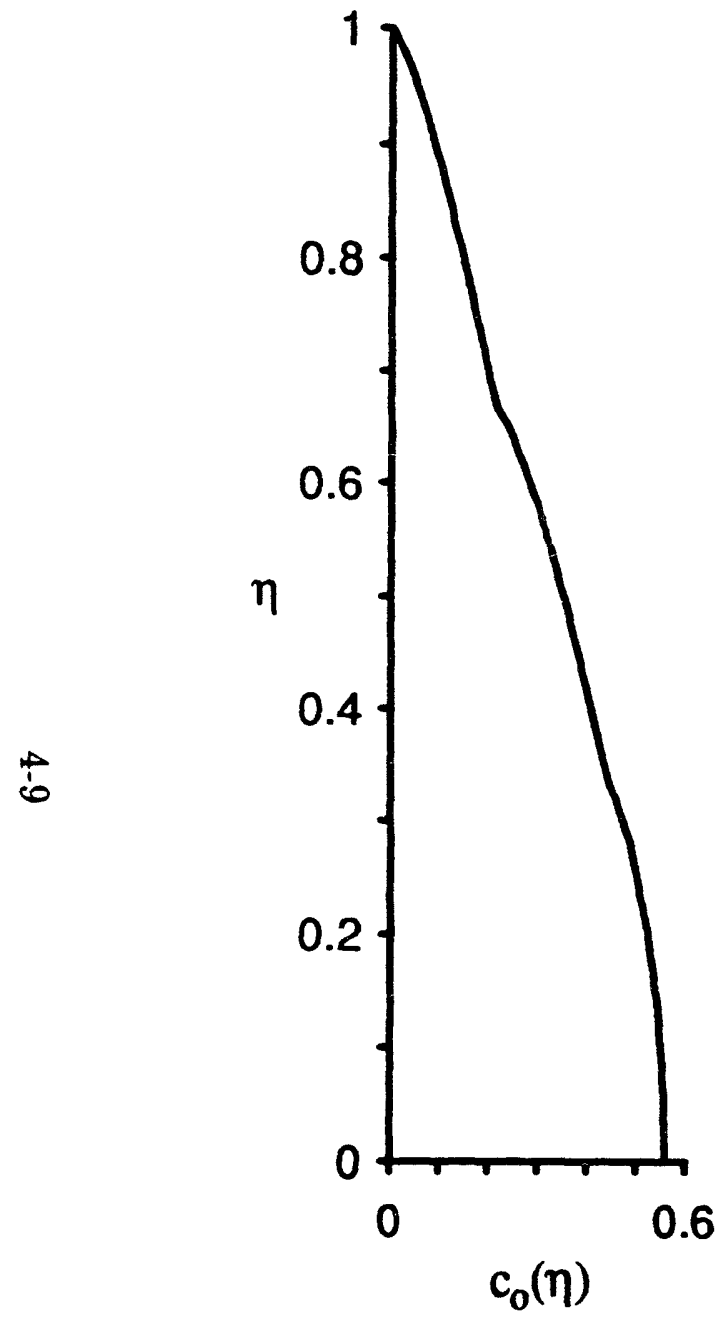

(a) Impulsive

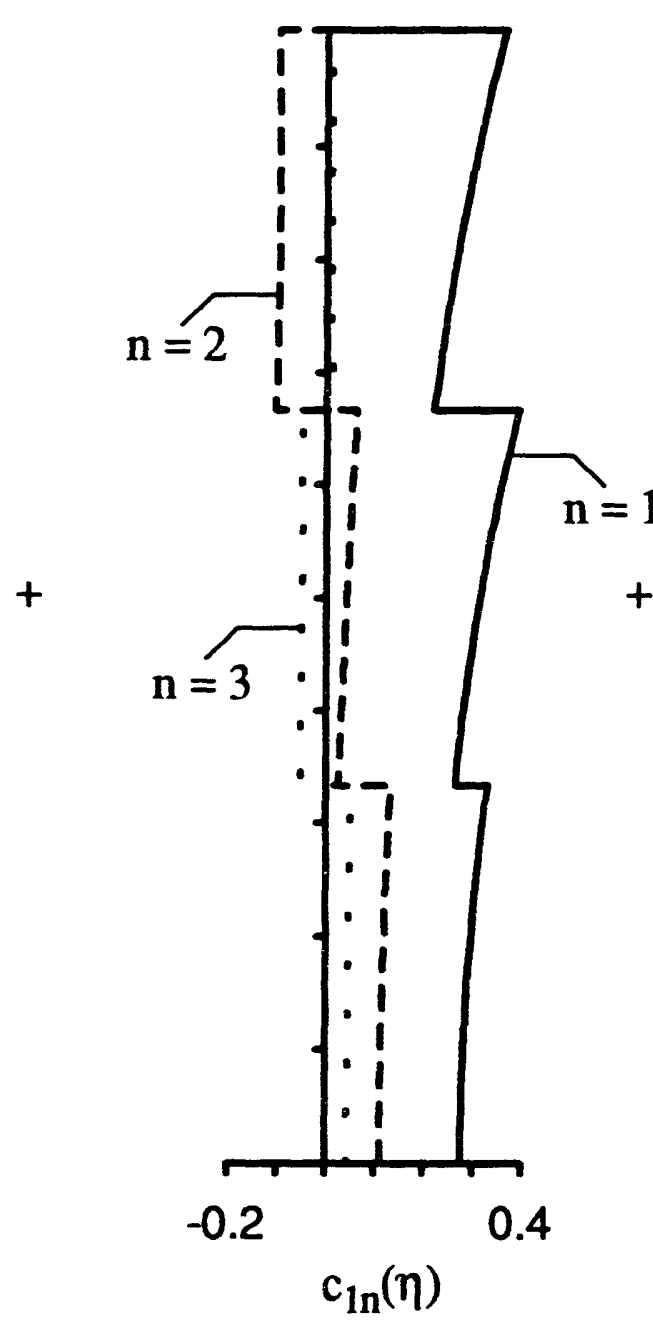

(b) Convective

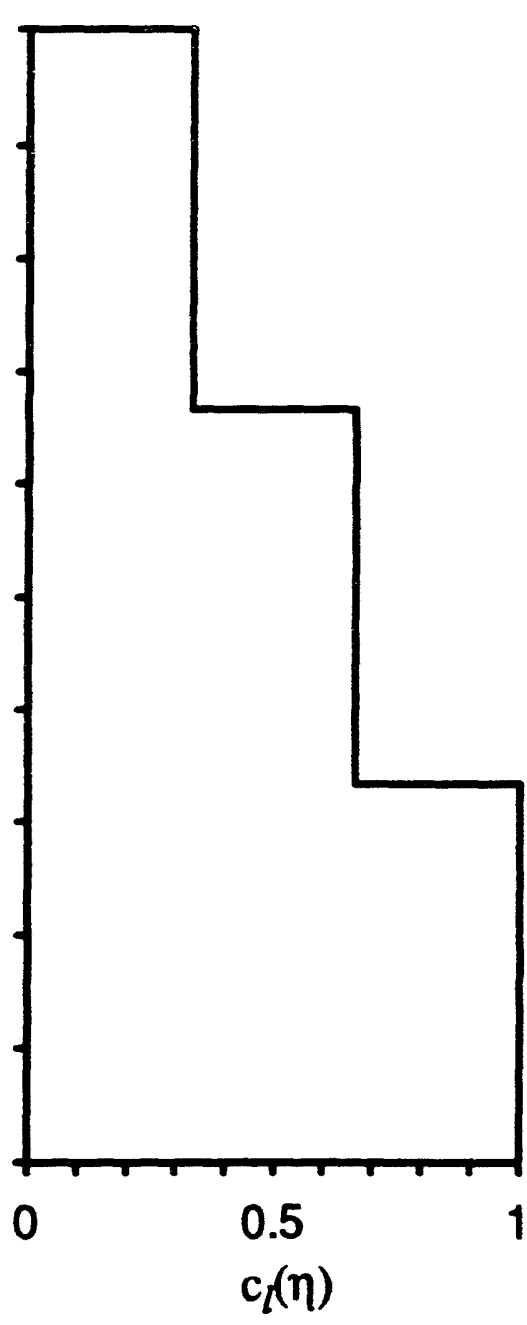

(c) Total

Figure 4.2 Interrelationship of coefficients for impulsive and convective components of wall pressure for three-layered system with $H / R=1, H_{1}=H_{2}=H_{3}=H / 3$ and $\rho_{3} / \rho_{2} / \rho_{1}=1 / 2 / 3$ 


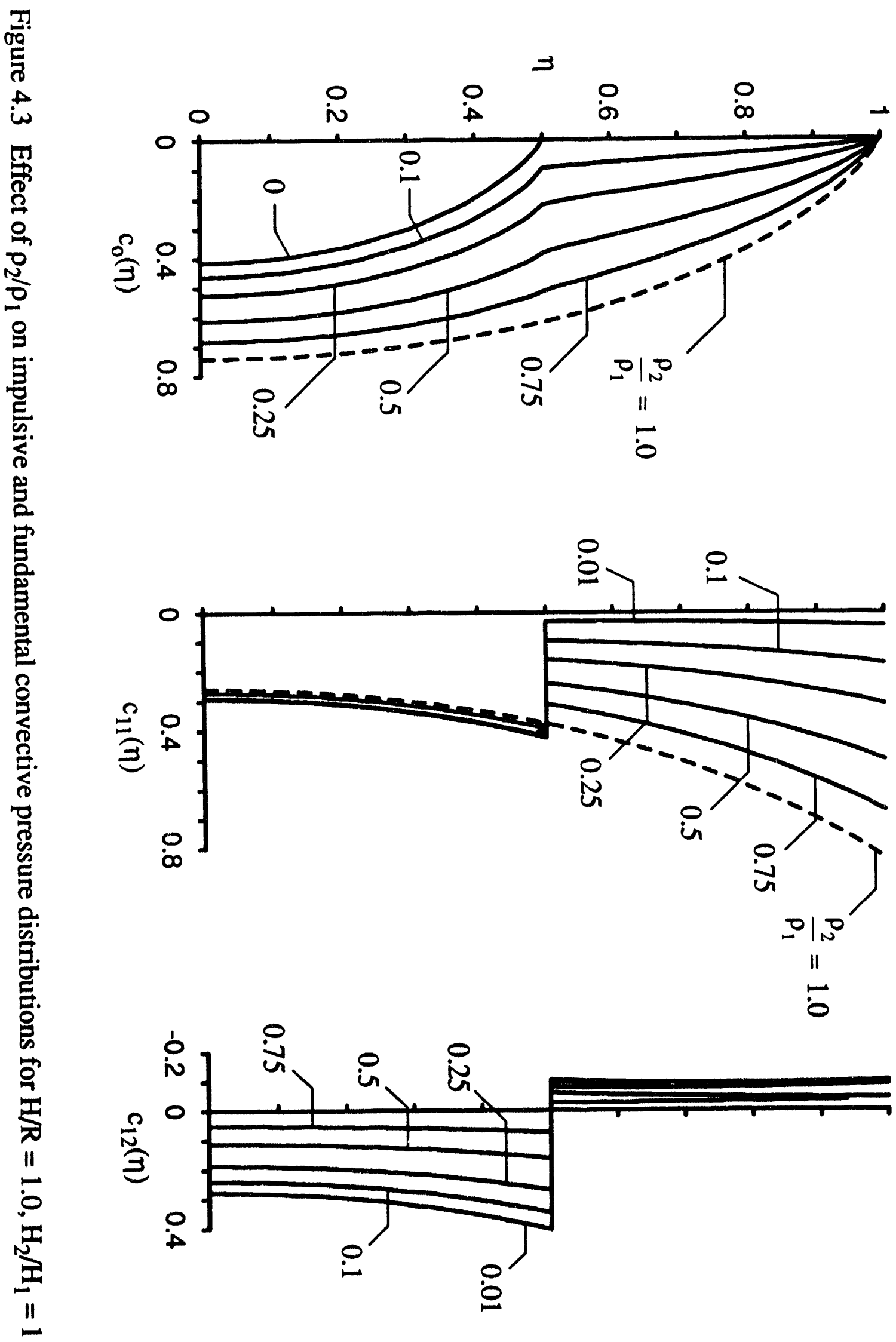




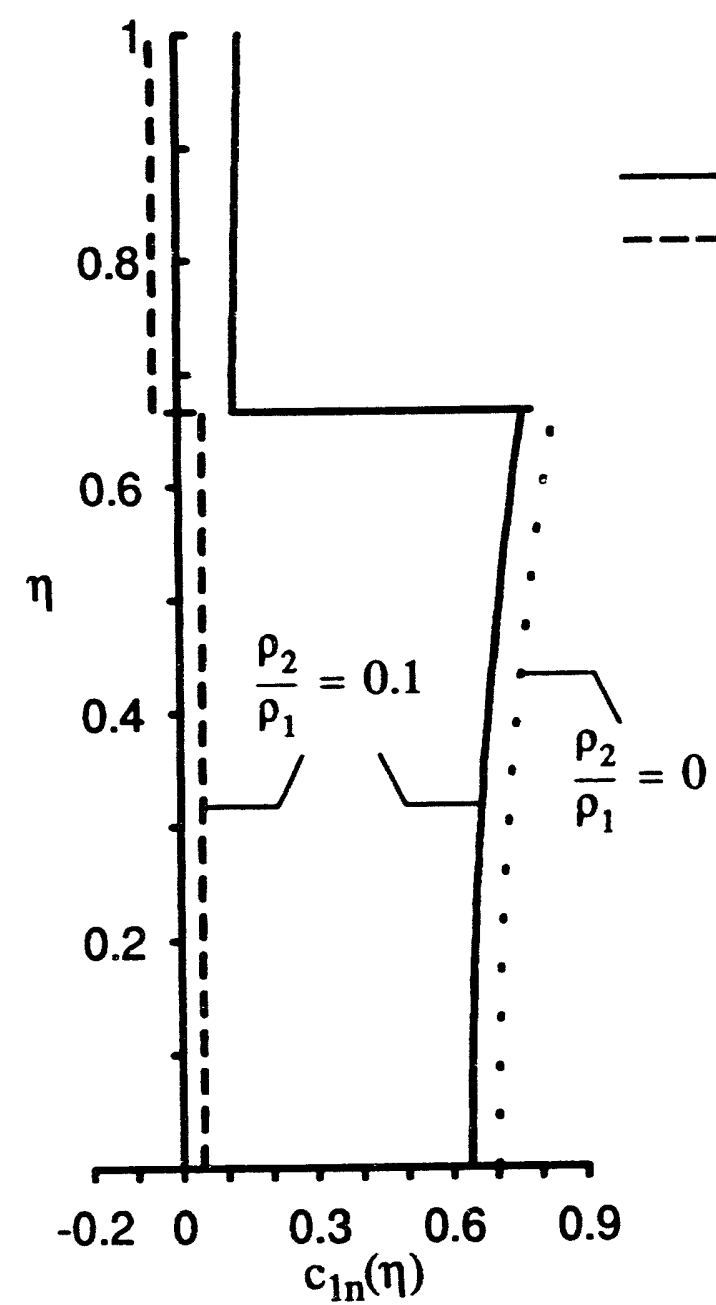

(a) for $\mathrm{H}_{2} / \mathrm{H}_{1}=0.5$

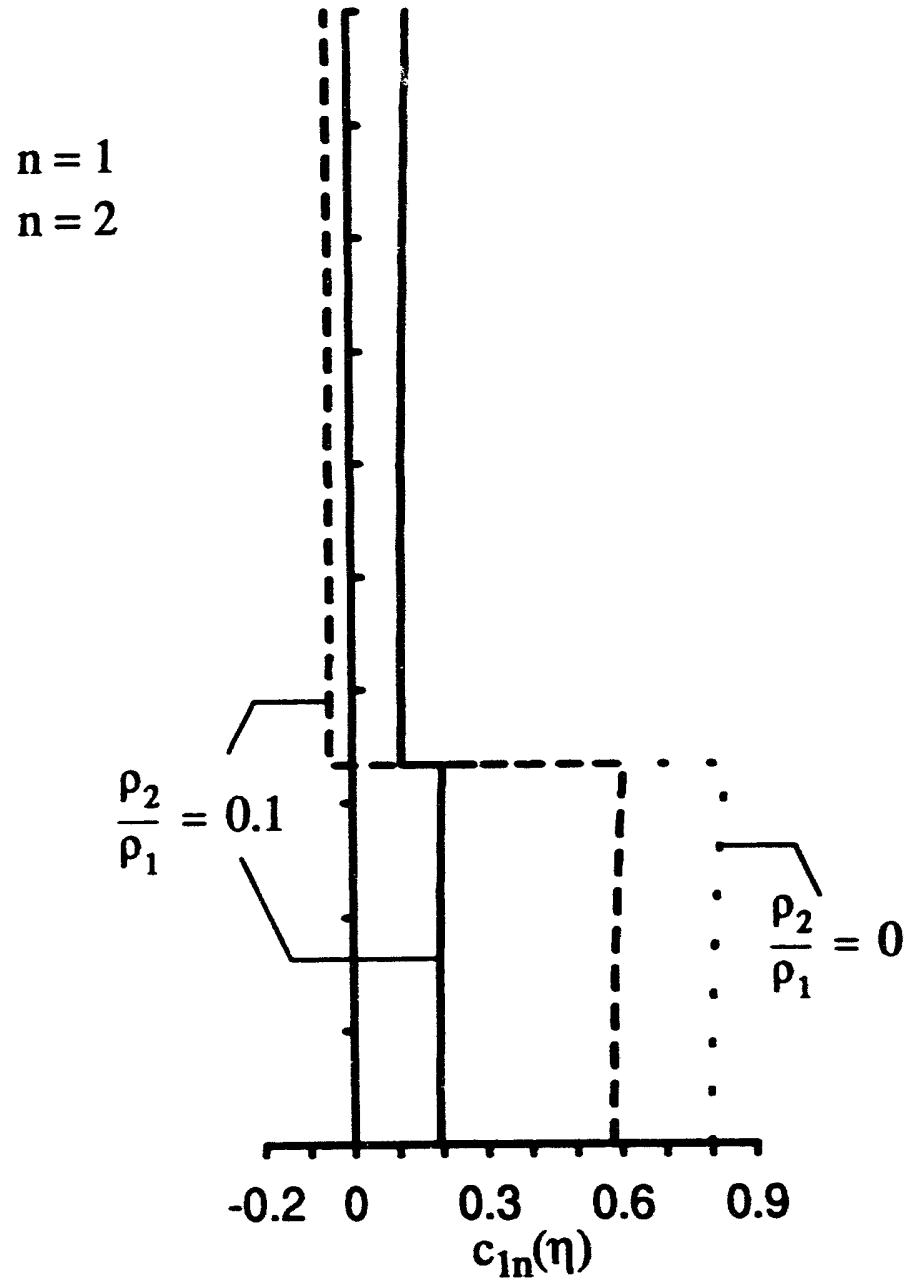

(b) for $\mathrm{H}_{2} / \mathrm{H}_{1}=2.0$

Figure 4.4 Effect of $H_{2} / H_{1}$ on convective pressure coefficient $c_{1 n}(\eta)$ as $\rho_{2} / \rho_{1}$ tends to zero; system with $H / R=1$ 


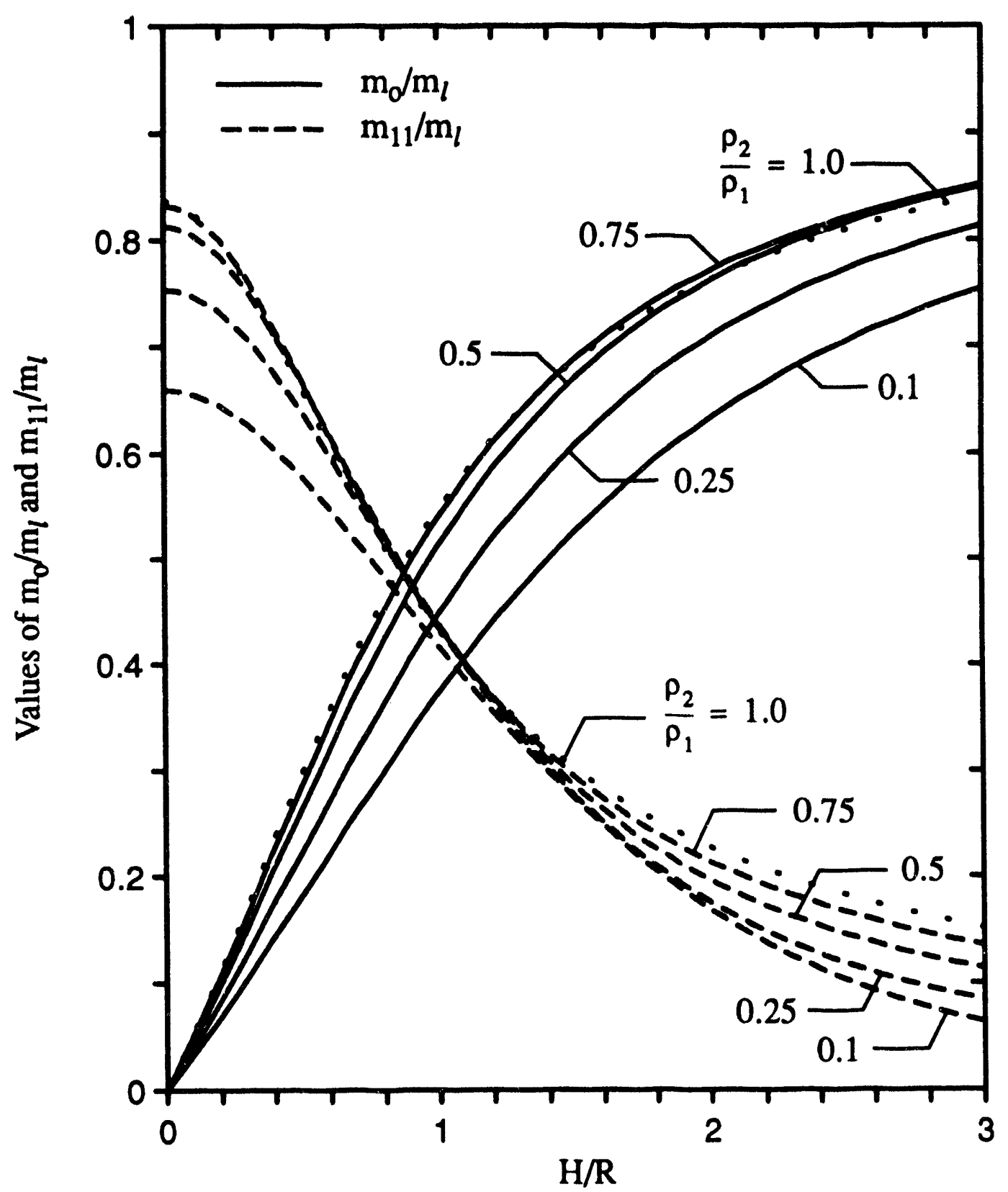

Figure 4.5 Impulsive and fundamental convective masses for two-layered systems with $\mathrm{H}_{2} / \mathrm{H}_{1}=1$ 


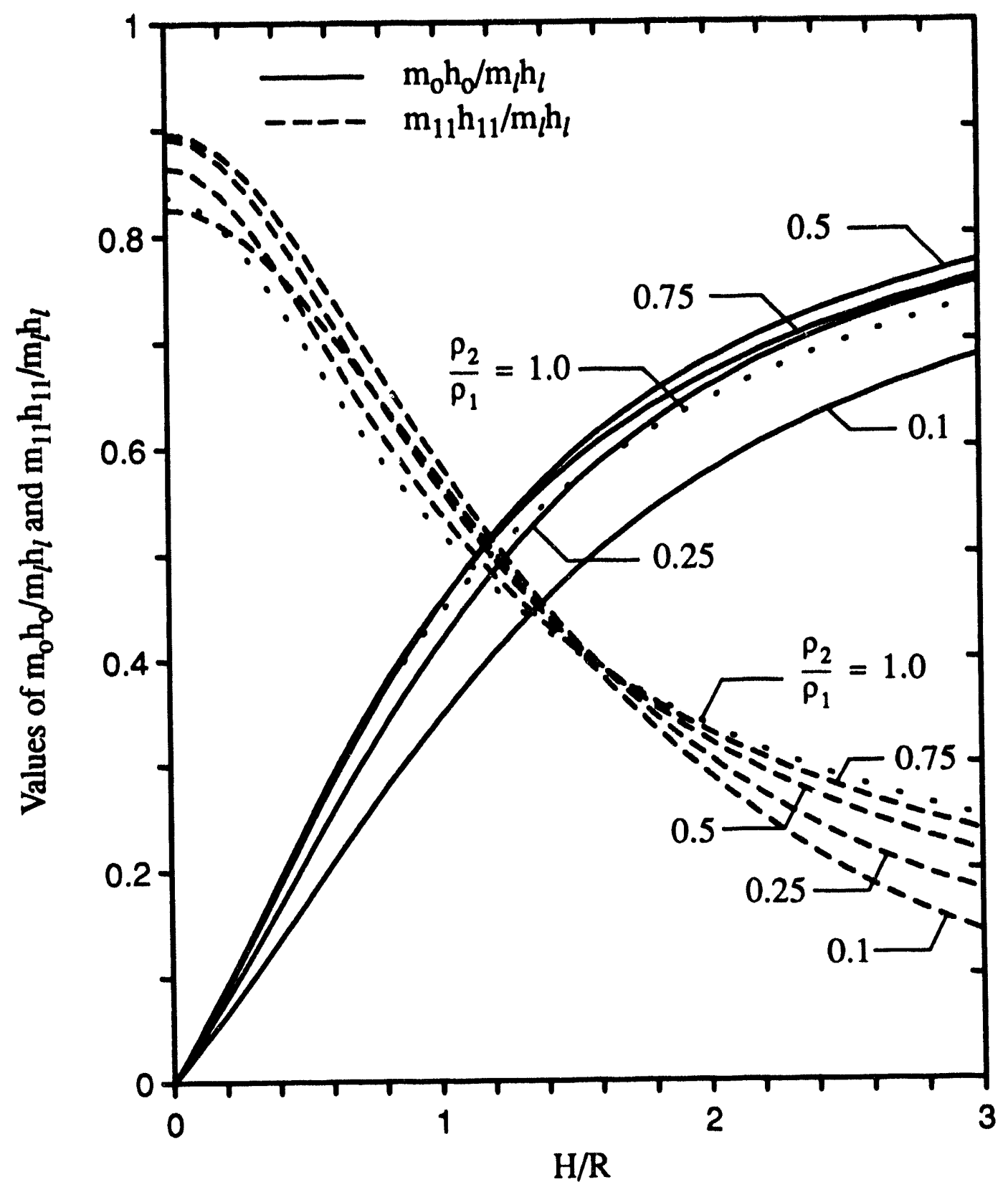

Figure 4.6 Normalized values of coefficients for impulsive and fundamental convective component of base moment for two-layered systems with $\mathrm{H}_{2} / \mathrm{H}_{1}=1$ 


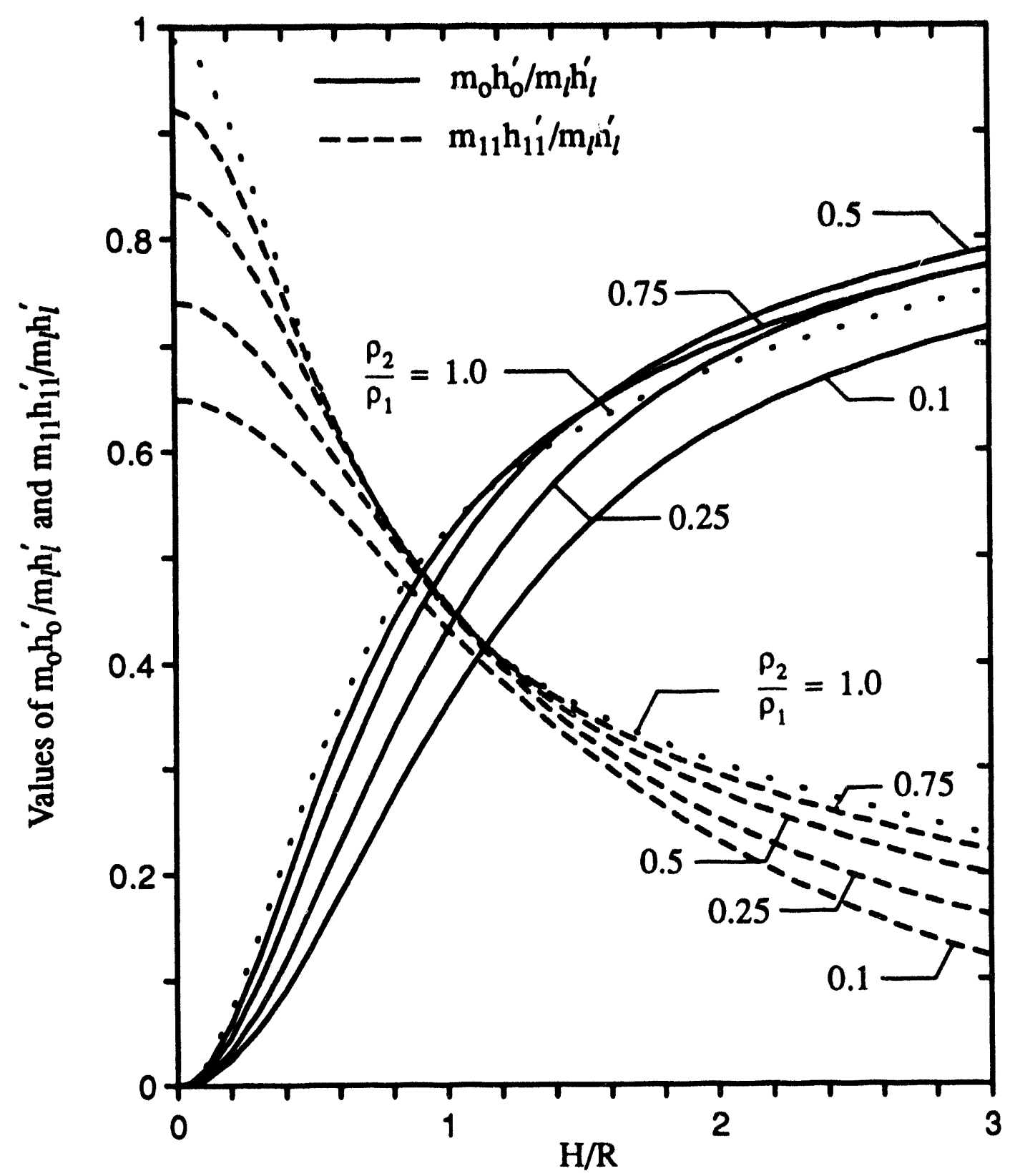

Figure 4.7 Normalized values of coefficients for impulsive and fundamental convective component of foundation moment for two-layered systems with $\mathrm{H}_{2} / \mathrm{H}_{1}=1$ 

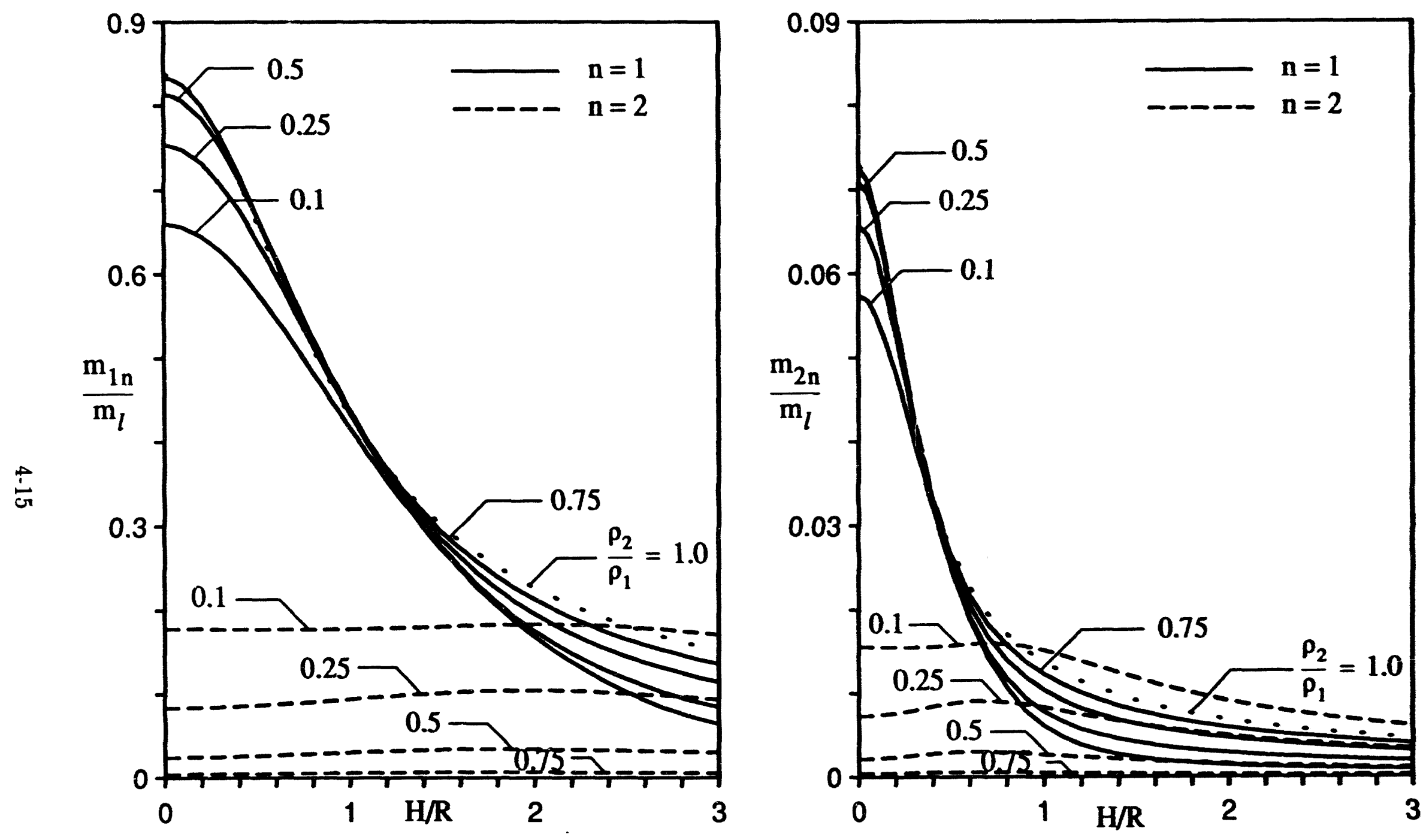

Figure 4.8 Convective masses for first two horizontal and two vertical modes of vibration of two-layered systems with $\mathrm{H}_{2} / \mathrm{H}_{1}=1$ 


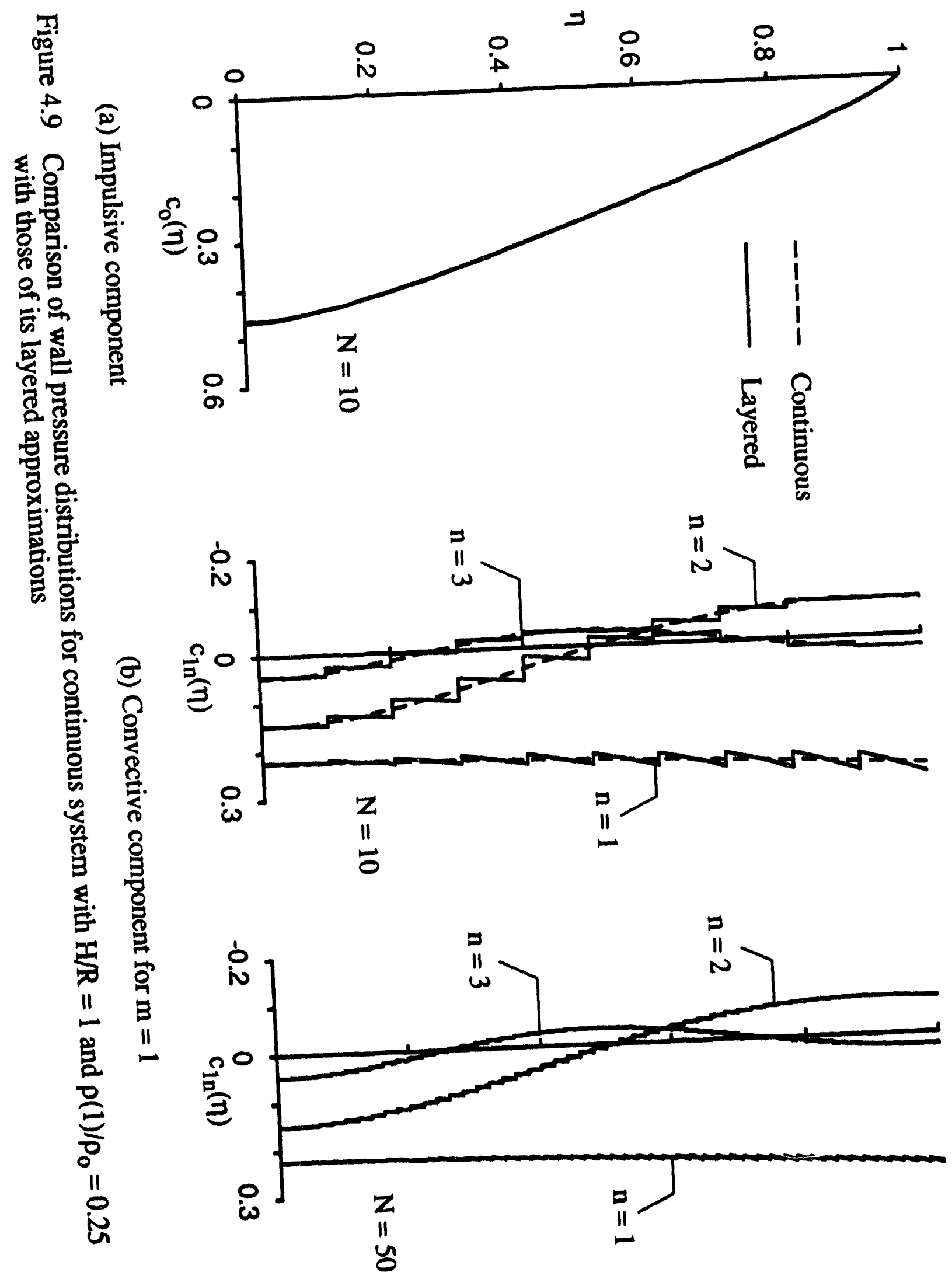


Table 4.1: Values of coefficients in expression for hydrodynamic wall pressure at selected sections of two-layered systems with different $H / R$ and $H_{2} / H_{1}$

\begin{tabular}{|c|c|c|c|c|c|c|c|c|c|c|c|}
\hline \multirow{2}{*}{$\frac{\rho_{2}}{\rho_{1}}$} & \multirow[b]{2}{*}{$\eta$} & \multicolumn{5}{|c|}{$H_{2} / H_{1}=0.5$} & \multicolumn{5}{|c|}{$H_{2} / H_{1}=2$} \\
\hline & & $c_{o}$ & $c_{11}$ & $c_{12}$ & $c_{21}$ & $c_{22}$ & $c_{o}$ & $c_{11}$ & $c_{12}$ & $c_{21}$ & $c_{22}$ \\
\hline \multirow{5}{*}{1} & & & & & & \multicolumn{2}{|c|}{$H / R=0.5$} & & & & \\
\hline & 1 & 0 & 0.837 & & 0.073 & & 0 & 0.837 & & 0.073 & \\
\hline & $I+$ & 0.270 & 0.687 & & 0.031 & & 0.378 & 0.603 & & 0.031 & \\
\hline & $I-$ & 0.270 & 0.687 & & 0.031 & & 0.378 & 0.603 & & 0.031 & \\
\hline & 0 & 0.411 & 0.575 & & 0.010 & & 0.411 & 0.575 & & 0.010 & \\
\hline \multirow[t]{4}{*}{0.75} & 1 & 0 & 0.672 & -0.045 & 0.059 & -0.004 & 0 & 0.667 & -0.040 & 0.057 & -0.002 \\
\hline & $I+$ & 0.218 & 0.557 & -0.046 & 0.025 & -0.005 & 0.304 & 0.492 & -0.046 & 0.012 & -0.006 \\
\hline & $I-$ & 0.218 & 0.692 & 0.028 & 0.033 & 0.005 & 0.304 & 0.550 & 0.105 & 0.014 & 0.010 \\
\hline & 0 & 0.380 & 0.580 & 0.024 & 0.011 & 0.002 & 0.352 & 0.525 & 0.100 & 0.010 & 0.007 \\
\hline \multirow[t]{4}{*}{0.5} & 1 & 0 & 0.495 & -0.076 & 0.044 & -0.008 & 0 & 0.486 & -0.068 & 0.040 & -0.004 \\
\hline & $I+$ & 0.158 & 0.415 & -0.077 & 0.019 & -0.009 & 0.219 & 0.369 & -0.076 & 0.009 & -0.009 \\
\hline & $I-$ & 0.158 & 0.702 & 0.054 & 0.036 & 0.010 & 0.219 & 0.474 & 0.237 & 0.014 & 0.023 \\
\hline & 0 & 0.347 & 0.588 & 0.045 & 0.012 & $0.00 ?$ & 0.286 & 0.453 & 0.226 & 0.010 & 0.016 \\
\hline \multirow[t]{4}{*}{0.25} & 1 & 0 & 0.291 & -0.082 & 0.027 & -0.009 & 0 & 0.283 & -0.074 & 0.023 & -0.005 \\
\hline & $I+$ & 0.086 & 0.248 & -0.081 & 0.012 & -0.009 & 0.120 & 0.223 & -0.079 & 0.006 & -0.008 \\
\hline & $I-$ & 0.086 & 0.726 & 0.069 & 0.043 & 0.015 & 0.120 & 0.347 & 0.424 & 0.012 & 0.040 \\
\hline & 0 & 0.310 & 0.608 & 0.058 & 0.014 & 0.005 & 0.212 & 0.331 & 0.405 & 0.009 & 0.028 \\
\hline \multirow[t]{5}{*}{0.1} & 1 & 0 & 0.139 & -0.056 & 0.014 & -0.007 & 0 & 0.134 & -0.051 & 0.011 & -0.004 \\
\hline & $I+$ & 0.038 & 0.121 & -0.054 & 0.006 & -0.006 & 0.051 & 0.109 & -0.052 & 0.003 & -0.005 \\
\hline & $I-$ & 0.038 & 0.765 & 0.054 & 0.054 & 0.013 & 0.051 & 0.202 & 0.609 & 0.009 & 0.055 \\
\hline & 0 & 0.286 & 0.641 & 0.046 & 0.018 & 0.004 & 0.163 & 0.192 & 0.581 & 0.006 & 0.039 \\
\hline & & & & & & HI & & & & & \\
\hline \multirow[t]{4}{*}{1} & 1 & 0 & 0.837 & & 0.073 & & 0 & 0.837 & & 0.073 & \\
\hline & $I+$ & 0.503 & 0.480 & & 0.012 & & 0.686 & 0.309 & & 0.002 & \\
\hline & $I-$ & 0.503 & 0.480 & & 0.012 & & 0.686 & 0.309 & & 0.002 & \\
\hline & 0 & 0.738 & 0.259 & & 0.001 & & 0.738 & 0.259 & & 0.001 & \\
\hline \multirow[t]{4}{*}{0.75} & 1 & 0 & 0.676 & .0 .048 & 0.057 & -0.003 & 0 & 0.660 & -0.033 & 0.055 & -0.001 \\
\hline & $1+$ & 0.416 & 0.392 & -0.055 & 0.010 & -0.007 & 0.565 & 0.254 & -0.056 & 0.002 & -0.007 \\
\hline & $I-$ & 0.416 & 0.505 & 0.042 & 0.013 & 0.008 & 0.565 & 0.297 & 0.110 & 0.002 & 0.010 \\
\hline & 0 & 0.701 & 0.272 & 0.023 & 0.001 & 0.001 & 0.652 & 0.249 & 0.093 & 0.001 & 0.003 \\
\hline
\end{tabular}




\begin{tabular}{|c|c|c|c|c|c|c|c|c|c|c|c|}
\hline \multirow{2}{*}{$\frac{\rho_{2}}{\rho_{1}}$} & \multirow[b]{2}{*}{$\eta$} & \multicolumn{5}{|c|}{$H_{2} / H_{1}=0.5$} & \multicolumn{5}{|c|}{$\mathrm{H}_{2} / \mathrm{H}_{1}=2$} \\
\hline & & $c_{0}$ & $c_{11}$ & $c_{12}$ & $c_{21}$ & $c_{22}$ & $c_{o}$ & $c_{11}$ & $c_{12}$ & $c_{21}$ & $c_{22}$ \\
\hline \multirow[t]{4}{*}{0.5} & 1 & 0 & 03 & -0.084 & 0.041 & -0.005 & 0 & 0.476 & -0.057 & 0.037 & -0.001 \\
\hline & It & 0.309 & 0.296 & $|-0.090|$ & 0.007 & -0.011 & 0.418 & 0.194 & -0.088 & 0.001 & -0.011 \\
\hline & $I-$ & 0.309 & 0.542 & 0.083 & 0.014 & 0.019 & 0.418 & 0.275 & 0.249 & 0.002 & 0.024 \\
\hline & 0 & 0.658 & 0.293 & 0.045 & 0.001 & 0.001 & 0.549 & 0.230 & 0.209 & 0.001 & 0.008 \\
\hline \multirow[t]{4}{*}{0.25} & 1 & 0 & 0.303 & -0.094 & 0.025 & -0.007 & 0 & 0.274 & -0.065 & 0.020 & -0.001 \\
\hline & It & 0.175 & 0.184 & -0.091 & 0.004 & -0.010 & 0.236 & 0.122 & -0.086 & 0.001 & -0.010 \\
\hline & $I-$ & 0.175 & 0.611 & 0.109 & 0.017 & 0.032 & 0.236 & 0.224 & 0.440 & 0.002 & 0.043 \\
\hline & 0 & 0.606 & 0.330 & 0.059 & 0.001 & 0.002 & 0.424 & 0.188 & 0.368 & 0.001 & 0.014 \\
\hline \multirow[t]{5}{*}{0.1} & 1 & 0 & 0.150 & -0.066 & 0.015 & -0.007 & 0 & 0.131 & -0.050 & 0.009 & -0.001 \\
\hline & $I+$ & 0.076 & 0.094 & -0.060 & 0.003 & -0.006 & 0.102 & 0.064 & -0.054 & 0.000 & -0.006 \\
\hline & $I-$ & 0.076 & 0.698 & 0.090 & 0.025 & 0.038 & 0.102 & 0.146 & 0.618 & 0.002 & 0.058 \\
\hline & 0 & 0.569 & 0.377 & 0.048 & 0.001 & 0.002 & 0.335 & 0.122 & 0.517 & 0.001 & 0.019 \\
\hline & & & & & & $H /$ & & & & & \\
\hline \multirow[t]{4}{*}{1} & 1 & 0 & 0.837 & & 0.073 & & 0 & 0.837 & & 0.073 & \\
\hline & $I+$ & 0.748 & 0.245 & & 0.002 & & 0.919 & 0.078 & & 0.000 & \\
\hline & $I-$ & 0.748 & 0.245 & & 0.002 & & $0 . y 19$ & 0.078 & & 0.000 & \\
\hline & 0 & 0.955 & 0.042 & & 0.000 & & 0.955 & 0.042 & & 0.000 & \\
\hline \multirow[t]{4}{*}{0.75} & 1 & 0 & 0.670 & -0.042 & 0.055 & -0.001 & 0 & 0.642 & -0.015 & 0.055 & -0.000 \\
\hline & It & 0.635 & 0.198 & -0.069 & 0.002 & -0.008 & 0.780 & 0.061 & -0.076 & 0.000 & -0.008 \\
\hline & $I-$ & 0.635 & 0.263 & 0.075 & 0.002 & 0.010 & 0.780 & 0.078 & 0.117 & 0.000 & 0.010 \\
\hline & 0 & 0.940 & 0.045 & 0.013 & 0.000 & 0.000 & 0.892 & 0.042 & 0.063 & 0.000 & 0.001 \\
\hline \multirow[t]{4}{*}{0.5} & 1 & 0 & 0.498 & -0.079 & 0.038 & -0.001 & 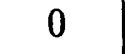 & 0.446 & -0.028 & 0.037 & -0.000 \\
\hline & It & 0.487 & 0.148 & -0.111 & 0.001 & -0.012 & 0.598 & 0.044 & -0.117 & 0.000 & -0.012 \\
\hline & $I-$ & 0.487 & 0.292 & 0.164 & 0.002 & 0.024 & 0.598 & 0.077 & 0.270 & 0.000 & 0.024 \\
\hline & 0 & 0.920 & 0.050 & 0.028 & 0.000 & 0.000 & 0.809 & 0.041 & 0.146 & 0.000 & 0.001 \\
\hline \multirow[t]{4}{*}{0.25} & 1 & 0 & 0.312 & -0.103 & 0.320 & -0.002 & 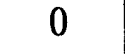 & 0.246 & -0.037 & 0.018 & -0.000 \\
\hline & $I+$ & 0.287 & 0.093 & -0.108 & 0.001 & -0.011 & 0.352 & 0.027 & -0.105 & 0.000 & -0.011 \\
\hline & $I-$ & 0.287 & 0.362 & 0.251 & 0.002 & 0.042 & 0.352 & 0.073 & 0.476 & 0.000 & 0.044 \\
\hline & . & 0.893 & 0.062 & 0.043 & 0.000 & 0.000 & 0.698 & 0.039 & 0.257 & 0.000 & 0.003 \\
\hline \multirow[t]{4}{*}{0.1} & 1 & 0 & 0.173 & -0.089 & 0.009 & -0.002 & 0 & 0.116 & -0.032 & 0.007 & -0 . \\
\hline & $I-$ & 0.128 & 0.052 & -0.068 & 0.000 & -0.006 & 0.157 & 0.015 & -0.059 & 0.000 & -0.006 \\
\hline & $I-$ & 0.128 & 0.4 & 0.247 & 0.003 & 0.0 & 0.157 & 0.062 & 0.647 & 0.000 & 0.060 \\
\hline & 0 & 0.872 & 0.084 & 0.042 & 0.000 & 0.000 & 0.611 & 0.033 & 0.349 & 0.000 & 0.003 \\
\hline
\end{tabular}


Table 4.2: Normalized values of effective masses in expression for base shear of two-layered systems with different $H / R$ and $H_{2} / H_{1}$

\begin{tabular}{|c|c|c|c|c|c|c|c|c|c|c|}
\hline \multirow[b]{2}{*}{$\frac{\rho_{2}}{\rho_{1}}$} & \multicolumn{5}{|c|}{$H_{2} / H_{1}=0.5$} & \multicolumn{5}{|c|}{$H_{2} / H_{1}=2$} \\
\hline & $\frac{\mathrm{m}_{\mathrm{o}}}{\mathrm{m}_{\ell}}$ & $\frac{m_{1}}{m_{\ell}}$ & $\frac{\mathrm{m}_{12}}{\mathrm{~m}_{\ell}}$ & $\frac{\mathrm{m}_{21}}{\mathrm{~m}_{\ell}}$ & $\frac{\mathrm{m}_{22}}{\mathrm{~m}_{\ell}}$ & $\frac{\mathrm{m}_{\ell}}{\mathrm{m}_{\ell}}$ & $\frac{m_{11}}{m_{\ell}}$ & $\frac{m_{12}}{m_{\ell}}$ & $\frac{\mathrm{m}_{21}}{\mathrm{~m}_{\ell}}$ & $\frac{\mathrm{m}_{22}}{\mathrm{~m}_{\ell}}$ \\
\hline & & & & & \multicolumn{2}{|c|}{$H / R=0.5$} & & & & \\
\hline 1 & 0.299 & 0.660 & & 0.027 & & 0.299 & 0.660 & & 0.027 & \\
\hline 0.75 & 0.288 & 0.670 & 0.002 & 0.027 & 0.000 & 0.289 & 0.663 & 0.008 & 0.026 & 0.001 \\
\hline 0.5 & 0.270 & 0.681 & 0.008 & 0.027 & 0.001 & 0.267 & 0.644 & 0.045 & 0.025 & 0.004 \\
\hline 0.25 & 0.241 & 0.694 & 0.018 & 0.029 & 0.003 & 0.218 & 0.551 & 0.176 & 0.022 & 0.014 \\
\hline 0.1 & 0.217 & 0.711 & 0.020 & 0.032 & 0.004 & 0.159 & 0.359 & 0.409 & 0.015 & 0.031 \\
\hline & & & & & \multicolumn{2}{|c|}{$H / R=1$} & & & & \\
\hline 1 & 0.547 & 0.432 & & 0.014 & & 0.547 & 0.432 & & 0.014 & \\
\hline 0.75 & 0.538 & 0.440 & 0.003 & 0.012 & 0.000 & 0.540 & 0.432 & 0.008 & 0.013 & 0.001 \\
\hline 0.5 & 0.517 & 0.453 & 0.012 & 0.011 & 0.002 & 0.512 & 0.421 & 0.046 & 0.011 & 0.003 \\
\hline 0.25 & 0.474 & 0.478 & 0.026 & 0.010 & 0.005 & 0.431 & 0.369 & 0.172 & 0.008 & 0.011 \\
\hline 0.1 & 0.433 & 0.512 & 0.029 & 0.010 & 0.008 & 0.322 & 0.252 & 0.384 & 0.005 & 0.023 \\
\hline & & & & & \multicolumn{2}{|c|}{$H / R=2$} & & & & \\
\hline 1 & 0.762 & 0.227 & & 0.007 & & 0.762 & 0.227 & & 0.007 & \\
\hline 0.75 & 0.769 & 6.217 & 0.004 & 0.006 & 0.000 & 0.768 & 0.214 & 0.007 & 0.006 & 0.000 \\
\hline 0.5 & 0.763 & 0.209 & 0.019 & 0.004 & 0.001 & 0.751 & 0.195 & 0.043 & 0.005 & 0.002 \\
\hline 0.25 & 0.732 & 0.209 & 0.048 & 0.003 & 0.004 & 0.674 & 0.158 & 0.153 & 0.003 & 0.006 \\
\hline 0.1 & 0.691 & 0.235 & 0.061 & 0.002 & 0.007 & 0.550 & 0.110 & 0.318 & 0.002 & 0.013 \\
\hline
\end{tabular}


Table 4.3: Normalized values of coefficients in expression for overturning moment at a section immediately above tank base of two-layered systems with different $H / R$ and $H_{2} / H_{1}$

\begin{tabular}{|c|c|c|c|c|c|c|c|c|c|c|}
\hline \multirow[b]{2}{*}{$\frac{\rho_{2}}{\rho_{1}}$} & \multicolumn{5}{|c|}{$H_{2} / H_{1}=0.5$} & \multicolumn{5}{|c|}{$H_{2} / H_{1}=2$} \\
\hline & $\frac{\mathrm{m}_{0} \mathrm{~h}_{\mathrm{o}}}{\mathrm{m}_{\ell} \mathrm{h}_{\ell}}$ & $\frac{\mathrm{m}_{11} \mathrm{~h}_{11}}{\mathrm{~m}_{\ell} \mathrm{h}_{\ell}}$ & $\frac{m_{12} h_{12}}{m_{\ell} h_{\ell}}$ & $\frac{m_{21} h_{21}}{m_{\ell} h_{\ell}}$ & $\frac{\mathrm{m}_{22} \mathrm{~h}_{22}}{\mathrm{~m}_{\ell} \mathrm{h}_{\ell}}$ & $\frac{m_{0} h_{0}}{m_{\ell} h_{\ell}}$ & $\frac{m_{\mu_{\mu}} h_{\mu}}{m_{\ell} h_{\ell}}$ & $\frac{m_{12} h_{12}}{m_{\ell} h_{\ell}}$ & $\frac{\mathrm{m}_{21} \mathrm{~h}_{21}}{\mathrm{~m}_{\ell} \mathrm{h}_{\ell}}$ & $\frac{\mathrm{m}_{22} \mathrm{~h}_{22}}{\mathrm{~m}_{\ell} \mathrm{h}_{\ell}}$ \\
\hline & & & & & \multicolumn{2}{|c|}{$H / R=0.5$} & & & & \\
\hline 1 & 0.238 & 0.703 & & 0.037 & & 0.238 & 0.703 & & 0.037 & \\
\hline 0.75 & 0.236 & 0.724 & -0.016 & 0.037 & -0.001 & 0.241 & 0.736 & -0.032 & 0.037 & -0.002 \\
\hline 0.5 & 0.227 & 0.746 & -0.028 & 0.038 & -0.002 & 0.239 & 0.772 & -0.064 & 0.038 & -0.004 \\
\hline 0.25 & 0.206 & 0.764 & -0.029 & 0.039 & -0.001 & 0.221 & 0.780 & -0.058 & 0.037 & -0.002 \\
\hline 0.1 & 0.181 & 0.768 & -0.016 & 0.041 & 0.001 & 0.177 & 0.643 & 0.110 & 0.032 & 0.011 \\
\hline & & & & & \multicolumn{2}{|c|}{$H / R=1$} & & & & \\
\hline 1 & 0.442 & 0.523 & & 0.022 & & 0.442 & 0.523 & & 0.022 & \\
\hline 0.75 & 0.446 & 0.538 & -0.016 & 0.021 & -0.001 & 0.453 & 0.542 & -0.028 & 0.022 & -0.001 \\
\hline 0.5 & 0.439 & 0.557 & -0.026 & 0.019 & 0.000 & 0.458 & 0.564 & -0.054 & 0.021 & -0.001 \\
\hline 0.25 & 0.407 & 0.583 & -0.023 & 0.017 & 0.004 & 0.434 & 0.573 & -0.040 & 0.018 & 0.003 \\
\hline 0.1 & 0.364 & 0.606 & -0.008 & 0.017 & 0.008 & 0.353 & 0.483 & 0.118 & 0.014 & 0.013 \\
\hline & & & & & \multicolumn{2}{|c|}{$H / R=2$} & & & & \\
\hline 1 & 0.644 & 0.337 & & 0.012 & & 0.644 & 0.337 & & 0.012 & \\
\hline 0.75 & 0.663 & 0.331 & -0.011 & 0.011 & 0.000 & 0.664 & 0.335 & -0.017 & 0.012 & -0.000 \\
\hline 0.5 & 0.670 & 0.325 & -0.011 & 0.009 & 0.001 & 0.679 & 0.330 & -0.028 & 0.011 & 0.000 \\
\hline 0.25 & 0.646 & 0.324 & 0.012 & 0.006 & 0.005 & 0.662 & 0.312 & 0.005 & 0.009 & 0.003 \\
\hline 0.1 & 0.595 & 0.346 & 0.037 & 0.004 & 0.010 & 0.569 & 0.259 & 0.144 & 0.006 & 0.011 \\
\hline
\end{tabular}


Table 4.4: Normalized values of coefficients in expression for foundation moment of two-layered systems with different $H / R$ and $H_{2} / H_{1}$

\begin{tabular}{|c|c|c|c|c|c|c|c|c|c|c|}
\hline \multirow[b]{2}{*}{$\frac{\rho_{2}}{\rho_{1}}$} & \multicolumn{5}{|c|}{$H_{2} / H_{1}=0.5$} & \multicolumn{5}{|c|}{$H_{2} / H_{1}=2$} \\
\hline & $\frac{\mathrm{m}_{0} \mathrm{~h}_{l}^{\prime}}{\mathrm{m}_{l} \mathrm{~h}_{\ell}^{\prime}}$ & $\frac{m_{11} h_{\mu 1}^{\prime}}{m_{\ell} h_{\ell}^{\prime}}$ & $\frac{\mathrm{m}_{12} \mathrm{~h}_{12}^{\prime}}{\mathrm{m}_{\ell} \mathrm{h}_{\ell}}$ & $\frac{\mathrm{m}_{21} \mathrm{~h}_{21}^{\prime}}{\mathrm{m}_{\ell} \mathrm{h}_{\ell}}$ & $\frac{\mathrm{m}_{22} \mathrm{~h}_{22}^{\prime}}{\mathrm{m}_{\ell} \mathrm{h}_{\ell}^{\prime}}$ & $\frac{m_{0} h_{o}^{\prime}}{m_{l} h_{l}^{\prime}}$ & $\frac{\mathrm{m}_{11} \mathrm{~h}_{\mu 1}^{\prime}}{\mathrm{m}_{\ell} \mathrm{h}_{\ell}^{\prime}}$ & $\frac{\mathrm{m}_{12} \mathrm{~h}_{12}^{\prime}}{\mathrm{m}_{\ell} \mathrm{h}_{\ell}^{\prime}}$ & $\frac{\mathrm{m}_{21} \mathrm{~h}_{21}^{\prime}}{\mathrm{m}_{\ell} \mathrm{h}_{l}^{\prime}}$ & $\frac{\mathrm{m}_{22} \mathrm{~h}_{22}^{\prime}}{\mathrm{m}_{\ell} \mathrm{h}_{\ell}^{\prime}}$ \\
\hline & & & & & \multicolumn{2}{|c|}{$H / R=0.5$} & & & & \\
\hline 1 & 0.292 & 0.687 & & 0.013 & & 0.292 & 0.687 & & 0.013 & \\
\hline 0.75 & 0.271 & 0.696 & 0.015 & 0.012 & -0.000 & 0.254 & 0.652 & 0.076 & 0.011 & 0.000 \\
\hline 0.5 & 0.244 & 0.708 & 0.032 & 0.011 & -0.000 & 0.204 & 0.586 & 0.195 & 0.009 & 0.001 \\
\hline 0.25 & 0.208 & 0.728 & 0.046 & 0.010 & 0.000 & 0.135 & 0.447 & 0.401 & 0.006 & 0.003 \\
\hline 0.1 & 0.185 & 0.759 & 0.040 & 0.010 & 0.001 & 0.088 & 0.265 & 0.633 & 0.004 & 0.006 \\
\hline & & & & & \multicolumn{2}{|c|}{$H / R=1$} & & & & \\
\hline 1.0 & 0.526 & 0.451 & & 0.015 & & 0.526 & 0.451 & & 0.015 & \\
\hline 0.75 & 0.521 & 0.459 & -0.000 & 0.013 & -0.000 & 0.509 & 0.445 & 0.025 & 0.013 & -0.000 \\
\hline 0.5 & 0.505 & 0.471 & 0.006 & 0.011 & 0.000 & 0.469 & 0.425 & 0.088 & 0.011 & -0.000 \\
\hline 0.25 & 0.467 & 0.494 & 0.020 & 0.009 & 0.002 & 0.372 & 0.362 & 0.245 & 0.007 & 0.002 \\
\hline 0.1 & 0.431 & 0.512 & 0.029 & 0.010 & 0.004 & 0.274 & 0.241 & 0.470 & 0.004 & 0.006 \\
\hline & & & & & \multicolumn{2}{|c|}{$H / R=2$} & & & & \\
\hline 1 & 0.676 & 0.305 & & 0.011 & & 0.676 & 0.305 & & 0.011 & \\
\hline 0.75 & 0.695 & 0.296 & -0.008 & 0.010 & 0.000 & 0.692 & 0.295 & -0.005 & 0.010 & -0.000 \\
\hline 0.5 & 0.704 & 0.285 & -0.005 & 0.008 & 0.001 & 0.696 & 0.278 & 0.009 & 0.009 & 0.000 \\
\hline 0.25 & 0.684 & 0.280 & 0.019 & 0.005 & 0.004 & 0.653 & 0.240 & 0.087 & 0.007 & 0.003 \\
\hline 0.1 & 0.645 & 0.296 & 0.040 & 0.003 & 0.008 & 0.559 & 0.174 & 0.247 & 0.004 & 0.007 \\
\hline
\end{tabular}


Table 4.5: Normalized values of effective masses in expression for base shear of three-layered systems with different $H / R$ and $H_{1}=H_{2}=H_{3}=H / 3$

\begin{tabular}{l|c|c|c|c|c|c|c}
\hline$\rho_{3} / \rho_{2} / \rho_{1}$ & $\frac{\mathrm{m}_{\ell}}{\mathrm{m}_{\ell}}$ & $\frac{\mathrm{m}_{11}}{\mathrm{~m}_{\ell}}$ & $\frac{\mathrm{m}_{12}}{\mathrm{~m}_{\ell}}$ & $\frac{\mathrm{m}_{13}}{\mathrm{~m}_{\ell}}$ & $\frac{\mathrm{m}_{21}}{\mathrm{~m}_{\ell}}$ & $\frac{\mathrm{m}_{22}}{\mathrm{~m}_{\ell}}$ & $\frac{\mathrm{m}_{23}}{\mathrm{~m}_{\ell}}$ \\
\hline & & & \multicolumn{2}{|c|}{$H / R=0.5$} & & & \\
$1 / 1 / 1$ & 0.299 & 0.660 & & & 0.027 & & \\
$1 / 2 / 3$ & 0.252 & 0.674 & 0.028 & 0.004 & 0.026 & 0.003 & 0.000 \\
$1 / 3 / 5$ & 0.229 & 0.672 & 0.044 & 0.008 & 0.027 & 0.005 & 0.001 \\
& & & \multicolumn{2}{|c|}{$H / R=1$} & & & \\
$1 / 1 / 1$ & 0.547 & 0.432 & & & 0.014 & & \\
$1 / 2 / 3$ & 0.495 & 0.450 & 0.032 & 0.004 & 0.010 & 0.003 & 0.001 \\
$1 / 3 / 5$ & 0.459 & 0.462 & 0.050 & 0.009 & 0.008 & 0.004 & 0.001 \\
& & & \multicolumn{2}{|c|}{$H / R=2$} & & & \\
$1 / 1 / 1$ & 0.762 & 0.227 & & & 0.007 & & \\
$1 / 2 / 3$ & 0.757 & 0.193 & 0.035 & 0.005 & 0.004 & 0.001 & 0.001 \\
$1 / 3 / 5$ & 0.731 & 0.189 & 0.058 & 0.012 & 0.003 & 0.002 & 0.001 \\
\hline
\end{tabular}


Table 4.6: Normalized values of coefficients in expression for overturning moment at a section immediately above tank base of three-layered systems with different $H / R$ and $H_{1}=H_{2}=H_{3}=H / 3$

\begin{tabular}{l|c|c|c|c|c|c|c}
\hline$\rho_{3} / \rho_{2} / \rho_{1}$ & $\frac{m_{0} h_{\ell}}{m_{\ell} h_{\ell}}$ & $\frac{m_{1} h_{14}}{m_{\ell} h_{\ell}}$ & $\frac{m_{12} h_{12}}{m_{\ell} h_{\ell}}$ & $\frac{m_{13} h_{13}}{m_{\ell} h_{\ell}}$ & $\frac{m_{21} h_{21}}{m_{\ell} h_{\ell}}$ & $\frac{m_{22} h_{22}}{m_{\ell} h_{\ell}}$ & $\frac{m_{23} h_{23}}{m_{\ell} h_{\ell}}$ \\
& & & \multicolumn{2}{|c|}{$H / R=0.5$} & & & \\
$1 / 1 / 1$ & 0.239 & 0.703 & & & 0.037 & & \\
$1 / 2 / 3$ & 0.226 & 0.789 & -0.057 & -0.010 & 0.039 & -0.003 & -0.001 \\
$1 / 3 / 5$ & 0.212 & 0.808 & -0.054 & -0.020 & 0.040 & 0.002 & -0.002 \\
& & & $H / R=1$ & & & \\
$1 / 1 / 1$ & 0.442 & 0.523 & \multicolumn{2}{|c|}{$H / R$} & & & \\
$1 / 2 / 3$ & 0.446 & 0.586 & -0.049 & -0.010 & 0.018 & 0.000 & -0.001 \\
$1 / 3 / 5$ & 0.424 & 0.609 & -0.041 & -0.020 & 0.017 & 0.002 & -0.001 \\
& & & $H / R=2$ & & & \\
$1 / 1 / 1$ & 0.644 & 0.337 & & & 0.012 & & \\
$1 / 2 / 3$ & 0.692 & 0.323 & -0.019 & -0.010 & 0.008 & 0.001 & -0.000 \\
$1 / 3 / 5$ & 0.682 & 0.321 & 0.001 & -0.018 & 0.006 & 0.003 & -0.000 \\
\hline
\end{tabular}


Table 4.7: Normalized values of coefficients in expression for foundation moment of three-layered systems with different $H / R$ and $H_{1}=H_{2}=H_{3}=H / 3$

\begin{tabular}{|c|c|c|c|c|c|c|c|}
\hline$\rho_{3} / \rho_{2} / \rho_{1}$ & $\frac{m_{0} h_{o}^{\prime}}{m_{\ell} h_{\ell}^{\prime}}$ & $\frac{\mathrm{m}_{11} \mathrm{~h}_{1,}^{\prime}}{\mathrm{m}_{\ell} \mathrm{h}_{\ell}^{\prime}}$ & $\frac{\mathrm{m}_{12} \mathrm{~h}_{12}^{\prime}}{\mathrm{m}_{\ell} \mathrm{h}_{\ell}^{\prime}}$ & $\frac{\mathrm{m}_{13} \mathrm{~h}_{13}^{\prime}}{\mathrm{m}_{\ell} \mathrm{h}_{\ell}^{\prime}}$ & $\frac{\mathrm{m}_{21} \mathrm{~h}_{21}^{\prime}}{\mathrm{m}_{\ell} \mathrm{h}_{\ell}^{\prime}}$ & $\frac{\mathrm{m}_{22} \mathrm{~h}_{22}^{\prime}}{\mathrm{m}_{\ell} \mathrm{h}_{\ell}^{2}}$ & $\frac{\mathrm{m}_{23} \mathrm{~h}_{23}^{\prime}}{\mathrm{m}_{\ell} \mathrm{h}_{\ell}^{\prime}}$ \\
\hline & & & \multicolumn{2}{|c|}{$H / R=0.5$} & & & \\
\hline $1 / 1 / 1$ & 0.292 & 0.687 & & 0.013 & & & \\
\hline $1 / 2 / 3$ & 0.196 & 0.639 & 0.113 & 0.040 & 0.008 & 0.001 & 0.000 \\
\hline \multirow[t]{2}{*}{$1 / 3 / 5$} & 0.168 & 0.643 & 0.113 & 0.063 & 0.008 & 0.001 & 0.000 \\
\hline & & & \multicolumn{2}{|c|}{$H / R=1$} & & & \\
\hline $1 / 1 / 1$ & 0.526 & 0.451 & & & 0.015 & & \\
\hline $1 / 2 / 3$ & 0.466 & 0.459 & 0.040 & 0.020 & 0.009 & 0.000 & -0.000 \\
\hline \multirow[t]{2}{*}{$1 / 3 / 5$} & 0.429 & 0.467 & 0.056 & 0.034 & 0.008 & 0.001 & -0.000 \\
\hline & & & \multicolumn{2}{|c|}{$H / R=2$} & & & \\
\hline $1 / 1 / 1$ & 0.678 & 0.305 & & & 0.011 & & \\
\hline $1 / 2 / 3$ & 0.715 & 0.271 & -0.000 & 0.001 & 0.007 & 0.001 & -0.000 \\
\hline $1 / 3 / 5$ & 0.702 & 0.263 & 0.020 & 0.003 & 0.005 & 0.002 & -0.000 \\
\hline
\end{tabular}


Table 4.8: Normalized values of effective masses in expression for base shear of a continuous system with $H / R=1, \rho(1) / \rho_{o}=0.25$ and its $\mathrm{N}$-layered approximation

\begin{tabular}{c|c|c|c|c|c|c|c}
\hline$N$ & $\frac{\mathrm{m}_{\ell}}{\mathrm{m}_{\ell}}$ & $\frac{\mathrm{m}_{1}}{\mathrm{~m}_{\ell}}$ & $\frac{\mathrm{m}_{12}}{\mathrm{~m}_{\ell}}$ & $\frac{\mathrm{m}_{13}}{\mathrm{~m}_{\ell}}$ & $\frac{\mathrm{m}_{21}}{\mathrm{~m}_{\ell}}$ & $\frac{\mathrm{m}_{22}}{\mathrm{~m}_{\ell}}$ & $\frac{\mathrm{m}_{23}}{\mathrm{~m}_{\ell}}$ \\
\hline 5 & 0.5022 & 0.4431 & 0.0338 & 0.0023 & 0.0099 & 0.0023 & 0.0001 \\
10 & 0.5007 & 0.4439 & 0.0340 & 0.0026 & 0.0099 & 0.0022 & 0.0001 \\
20 & 0.5004 & 0.4441 & 0.0341 & 0.0027 & 0.0099 & 0.0022 & 0.0001 \\
30 & 0.5003 & 0.4442 & 0.0341 & 0.0027 & 0.0099 & 0.0022 & 0.0001 \\
50 & 0.5003 & 0.4442 & 0.0341 & 0.0027 & 0.0099 & 0.0022 & 0.0001 \\
Cont. System & 0.5003 & 0.4442 & 0.0341 & 0.0027 & 0.0099 & 0.0021 & 0.0001 \\
\hline
\end{tabular}

Table 4.9: Normalized values of coefficients in expression for overturning moment at a section immediately above tank base for a continuous system with $H / R=1, \rho(1) / \rho_{o}=0.25$ and its $\mathrm{N}$-layered approximation

\begin{tabular}{c|c|c|c|c|c|c|c}
\hline$N$ & $\frac{m_{0} h_{\ell}}{m_{\ell} h_{\ell}}$ & $\frac{m_{1} h_{11}}{m_{\ell} h_{\ell}}$ & $\frac{m_{12} h_{12}}{m_{\ell} h_{\ell}}$ & $\frac{m_{13} h_{13}}{m_{\ell} h_{\ell}}$ & $\frac{m_{21} h_{21}}{m_{\ell} h_{\ell}}$ & $\frac{m_{22} h_{22}}{m_{\ell} h_{\ell}}$ & $\frac{m_{23} h_{23}}{m_{\ell} h_{\ell}}$ \\
\hline 5 & 0.4557 & 0.5861 & -0.0645 & -0.0023 & 0.0192 & -0.0019 & -0.0002 \\
10 & 0.4565 & 0.5896 & -0.0669 & -0.0026 & 0.0193 & -0.0021 & -0.0002 \\
20 & 0.4567 & 0.5905 & -0.0675 & -0.0026 & 0.0193 & -0.0022 & -0.0002 \\
30 & 0.4567 & 0.5907 & -0.0676 & -0.0027 & 0.0193 & -0.0022 & -0.0002 \\
50 & 0.4568 & 0.5908 & -0.0676 & -0.0027 & 0.0193 & -0.0022 & -0.0002 \\
Cont. System & 0.4568 & 0.5908 & -0.0677 & -0.0027 & 0.0193 & -0.0022 & -0.0002 \\
\hline
\end{tabular}


Table 4.10: Normalized values of coefficients in expression for foundation moment of a continuous system with $H / R=1, \rho(1) / \rho_{o}=0.25$ and its N-layered approximation

\begin{tabular}{c|c|c|c|c|c|c|c}
\hline$N$ & $\frac{\mathrm{m}_{o} \mathrm{~h}_{\ell}^{\prime}}{\mathrm{m}_{\ell} \mathrm{h}_{\ell}^{\prime}}$ & $\frac{m_{11} \mathrm{~h}_{\mu 1}^{\prime}}{\mathrm{m}_{\ell} \mathrm{h}_{\ell}^{\prime}}$ & $\frac{\mathrm{m}_{12} \mathrm{~h}_{12}^{\prime}}{\mathrm{m}_{\ell} \mathrm{h}_{\ell}^{\prime}}$ & $\frac{\mathrm{m}_{13} \mathrm{~h}_{13}^{\prime}}{\mathrm{m}_{\ell} \mathrm{h}_{\ell}^{\prime}}$ & $\frac{\mathrm{m}_{21} \mathrm{~h}_{21}^{\prime}}{\mathrm{m}_{\ell} \mathrm{h}_{\ell}^{\prime}}$ & $\frac{\mathrm{m}_{22} \mathrm{~h}_{22}^{\prime}}{\mathrm{m}_{\ell} \mathrm{h}_{\ell}^{\prime}}$ & $\frac{\mathrm{m}_{23} \mathrm{~h}_{23}^{\prime}}{\mathrm{m}_{\ell} \mathrm{h}_{\ell}^{\prime}}$ \\
\hline 5 & 0.4541 & 0.4390 & 0.0619 & 0.0222 & 0.0095 & -0.0005 & 0.0000 \\
10 & 0.4391 & 0.4276 & 0.0651 & 0.0277 & 0.0092 & -0.0005 & 0.0001 \\
20 & 0.4313 & 0.4208 & 0.0653 & 0.0289 & 0.0090 & -0.0005 & 0.0001 \\
30 & 0.4287 & 0.4184 & 0.0652 & 0.0290 & 0.0090 & -0.0005 & 0.0001 \\
50 & 0.4266 & 0.4164 & 0.0650 & 0.0290 & 0.0090 & -0.0005 & 0.0001 \\
Cont. System & 0.4234 & 0.4133 & 0.0646 & 0.0289 & 0.0089 & -0.0005 & 0.0001 \\
\hline
\end{tabular}




\section{SECTION 5}

\section{CONCLUSIONS}

With the information presented herein, the response to horizontal base shaking of rigid, vertical, circular cylindrical tanks containing an arbitrary number of uniform liquid layers of varying thicknesses and densities may be evaluated rationally and effectively. The comprehensive numerical solutions that have been presented elucidate the underlying response mechanisms, as well as the effects and relative importance of the numerous parameters involved. The principal conclusions of the study are as follows :

1. The response of an $N$-layered system may be expressed as the sum of an impulsive component and an infinite number of horizontal convective or sloshing components, each associated with $N$ vertical modes of vibration.

2. The $n$th vertical mode for each horizontal mode of vibration exhibits $(n-1)$ changes in sign. These changes are due to the in-phase or out-of-phase sloshing actions of the interfaces.

3. The impulsive pressure component is continuous and increases from zero at the top to a maximum at the base, whereas the convective pressure components are discontinuous at the layer interfaces, the magnitude of the discontinuity being a function of the tank proportions and of the relative densities and thicknesses of the layers.

4. When normalized with respect to the pressures computed on the assumption that the entire liquid acts as a rigic mass, the coefficients for the impulsive and all convective components of the hydrodynamic wall pressures add up to unity. The same is also true of the corresponding coefficients for base shear and base moments in the tank.

5. The impulsive component of response may be evaluated either as the difference between the response computed on the assumption that the entire liquid acts as a rigid mass and the sum of all convective components of response or, independently, without the prior evaluation of the convective effects.

6. For two-layered systems with ratios of mass densities in the range between 0.5 and 1.0, the base shear and base moments may be related simply to those obtained from well-established solutions for homogeneous systems. 
7. The solutions for layered systems presented herein may also be used to accurately evaluate the responses of systems with arbitrary and continuous variations in liquid density. 


\section{SECTION 6}

\section{APPENDIX I}

\subsection{Derivation of Eq. (23)}

On substituting Eqs. (7) and (16) for $\left\{D_{m}(t)\right\}$ and $\left\{\ddot{D}_{m}(t)\right\}$ into Eq. (5), one obtains

$$
\begin{gathered}
{[\mathcal{A}] R\left(\sum_{n=1}^{N} \omega_{m n}^{2}\left\{d_{m n}\right\}\right) \frac{\ddot{x}_{g}(t)}{g}-[\mathcal{A}] R\left(\sum_{n=1}^{N} \omega_{m n}^{2}\left\{d_{m n}\right\} \frac{A_{m n}(t)}{g}\right)} \\
+[\mathcal{B}] \lambda_{m} g\left(\sum_{n=1}^{N}\left\{d_{m n}\right\} \frac{A_{m n}(t)}{g}\right)=\epsilon_{m} \lambda_{m}\{s\} \ddot{x}_{g}(t)
\end{gathered}
$$

which on further dividing through by $\lambda_{m}$, grouping the terms with similar temporal variations, and making use of Eqs. (10) and (21), can be written in the form

$$
\left([\mathcal{A}]\left\{e_{m}\right\}-\epsilon_{m}\{s\}\right) \ddot{x}_{g}(t)=\sum_{n=1}^{N}\left(C_{m n}^{2}[\mathcal{A}]\left\{d_{m n}\right\}-[\mathcal{B}]\left\{d_{m n}\right\}\right) A_{m n}(t)
$$

Since the temporal variations of the two members of the latter equation are different, the equation can hold true only if the terms in parentheses on either side of the equation are equal to zero. On equating the left-hand member to zero, one obtains Eq. (23), and on doing the same with each of the right-hand members, one obtains the additional relation,

$$
C_{m n}^{2}[\mathcal{A}]\left\{d_{m n}\right\}=[\mathcal{B}]\left\{d_{m n}\right\}
$$




\section{SECTION 7}

\section{APPENDIX II}

\subsection{Derivation of Eq. (51)}

From Eq. (20) and the expressions for the elements of the matrix $[\mathcal{A}]$ given in Reference 1 , the difference in the convective pressure coefficients across the $j$ th interface may be written as

$$
\left[\frac{\rho_{j}}{\rho_{1}} c_{m n, j}\left(\alpha_{j}\right)-\frac{\rho_{j+1}}{\rho_{1}} c_{m n, j+1}(0)\right]=C_{m n}^{2}\left[\mathcal{A}_{j, j-1} d_{m n, j-1}+\mathcal{A}_{j, j} d_{m n, j}+\mathcal{A}_{j, j+1} d_{m n, j+1}\right]
$$

On applying Eq. (60) to each of the $N$ interfaces and normalizing the results in the form of Eq. (49), the difference in the interfacial values of the convective pressure coefficients can be written in vectorial form as

$$
\left\{c_{m n}^{-}-c_{m n}^{+}\right\}=C_{m n}^{2}[\mathcal{A}]\left\{d_{m n}\right\}
$$

and, by virtue of Eq. (59), as

$$
\left\{c_{m n}^{-}-c_{m n}^{+}\right\}=[\mathcal{B}]\left\{d_{m n}\right\}
$$

On summing the latter expression over $n$, making use of Eq. (11) and the fact that $\operatorname{diag}[B]=\{s\}$, one obtains the desired Eq. (51). Finally, on summing Eq. (51) over $m$ and making use of Eq. (12), one obtains the additional relation

$$
\sum_{m=1}^{\infty} \sum_{n=1}^{N}\left\{c_{m n}^{-}-c_{m n}^{+}\right\}=\{s\}
$$




\section{SECTION 8}

\section{REFERENCES}

1. A. S. Veletsos, and P. Shivakumar, 'Sloshing response of layered liquids in rigid tanks', Journal of Earthquake Engineering and Structural Dynamics Vol. 22, 801821 (1993).

2. Y. Tang, and Y. W. Chang, 'Dynamic response of tank containing two liquids', Rep. $A N L / R E-92 / 1$, Argonne Nat. Lab., Argonne, Ill. (1992).

3. Y. Tang, 'Dynamic response of tank containing two liquids', Journal of Engineering Mechanics, ASCE, Vol. 119, No. 3, 531-548 (1993).

4. Y. Tang, and Y. W. Chang, 'The exact solutions to the dynamic response of tanks containing two liquids, Rep. ANL/RE-93/2, Argonne Nat. Lab., Argonne, Ill. (1993).

5. Y. Tang, 'Sloshing displacements in a tank containing two liquids, Proc. ASME, PVP Conf., 1993, Vol. PVP. 259, pp. 179-184

6. A. S. Veletsos, and P. Shivakumar, Discussion of 'Dynamic response of tank containing two liquids' by Y. Tang, to appear in Journal of Engineering Mechanics, ASCE

7. Y.W. Chang, Discussion of 'Sloshing response of layered liquids in rigid tanks' by A. S. Veletsos and P. Shivakumar, to appear in Journal of Earthquake Engineering and Structural Dynamics.

8. A. S. Veletsos, and P. Shivakumar, 'Reply to discussion by Y. W. Chang', to appear in Journal of Earthquake Engineering and Structural Dynamics.

9. A. S. Veletsos, 'Seismic response and design of liquid storage Tanks', Guidelines for the Seismic Design of Oil and Gas Pipeline Systems, Technical Council on Lifeline Earthquake Engineering, ASCE, New York, 1984, pp. 255-370 and 443-461 


\section{SECTION 9}

\section{NOTATION}

[A] tri-diagonal, symmetric matrix of size $N \times N$

$A_{m n}(t)$ instantaneous pseudoacceleration for $m$ th horizontal and $n$th vertical mode of vibration

$A_{m n} \quad$ maximum value of $A_{m n}(t)$

[B] diagonal matrix of size $N \times N$

$c_{o, j}$ dimensionless coefficient in expression for impulsive pressure in $j$ th layer, given by Eq. (26)

$c_{m n, j}$ dimensionless coefficient in expression for convective component of pressure in $j$ th layer associated with $m$ th horizontal and $n$th vertical mode of vibration, given by Ea. (20)

$C_{m n} \quad$ dimensionless coefficient in expression for $\omega_{m n}$

$\left\{d_{m n}\right\} \quad$ vector of displacement coefficients in expression for $\left\{D_{m}\right\}$, given by Eq. (8)

$\left\{D_{m}\right\} \quad$ vector of interfacial vertical displacements of liquid along the tank wall when the system is vibrating in its $m$ th horizontal mode of vibration

$\left\{\hat{D}_{m n}\right\} \quad$ vector of amplitudes of interfacial vertical displacements for the $m$ th horizontal and $n$th vertical natural mode of vibration

$\left\{e_{m}\right\} \quad$ vector of dimensionless coefficients in expressions for impulsive effects, defined by Eq. (21) and evaluated from Eq. (23)

$e_{m, j} \quad j$ th element of $\left\{e_{m}\right\}$

$g \quad$ acceleration due to gravity

$h_{l} \quad$ height of center of gravity of liquid mass from tank base

$h_{o} \quad$ height of impulsive mass $m_{o}$ from tank base

$h_{m n} \quad$ height of convective mass $m_{m n}$ from tank base

$H \quad$ total depth of liquid in tank

$H_{j} \quad$ thickness of $j$ th liquid layer

$J_{1} \quad$ Bessel function of first kind and first order

$L_{j} \quad$ height of $j$ th liquid interface from tank base

$m_{l} \quad$ total liquid mass in tank

$m_{o} \quad$ impulsive component of liquid mass, given by Eq. (34) 


\begin{tabular}{|c|c|}
\hline$m_{m n}$ & $\begin{array}{l}\text { convective component of liquid mass associated with } m \text { th horizontal and } \\
n \text {th vertical mode of vibration, given by Eq. (35) }\end{array}$ \\
\hline$M(t)$ & $\begin{array}{l}\text { instantaneous value of overturning moment at a section just above the tank } \\
\text { base, given by Eq. (39) }\end{array}$ \\
\hline$M^{\prime}(t)$ & instantaneous value of foundation moment, given by Eq. (44) \\
\hline$N$ & number of superposed liquid layers of different densities \\
\hline$p_{j}$ & hydrodynamic pressure in $j$ th liquid layer, given by Eq. (17) \\
\hline$p_{j}^{i}$ & $\begin{array}{l}\text { impulsive component of hydrodynamic pressure in } j \text { th liquid layer, given } \\
\text { by Eq. (18) }\end{array}$ \\
\hline$p_{j}^{c}$ & $\begin{array}{l}\text { convective component of hydrodynamic pressure in } j \text { th liquid layer, given } \\
\text { by Eq. (19) }\end{array}$ \\
\hline$Q_{b}(t)$ & instantaneous value of base shear, given by Eq. (33) \\
\hline$R$ & radius of cylindrical tank \\
\hline$\{s\}$ & $\begin{array}{l}\text { a vector of size } N \text {, the elements of which are the same as the diagonal } \\
\text { elements of }[\mathcal{B}]\end{array}$ \\
\hline$t$ & time \\
\hline$U_{m n}(t)$ & $\begin{array}{l}\text { instantaneous deformation of a simple oscillator with a natural frequency } \\
\text { equal to that of the } m \text { th horizontal and } n \text {th vertical mode of vibration }\end{array}$ \\
\hline$\ddot{x}_{g}(t)$ & instantaneous value of free-field ground acceleration \\
\hline$z$ & vertical distance measured from tank base \\
\hline$z_{j}$ & vertical coordinate within the $j$ th liquid layer \\
\hline$\alpha_{j}$ & $=H_{j} / R=$ ratio of layer height to radius of tank \\
\hline$\beta$ & positive decay factor defining exponential variation in liquid density \\
\hline$\gamma_{1}$ & unit weight of lower-most or bottom layer \\
\hline$\epsilon_{m}$ & dimensionless factor defined by Eq. (6) \\
\hline$\eta$ & $=z / H=$ normalized vertical distance measured from tank base \\
\hline$\eta_{j}$ & $=z_{j} / R=$ normalized vertical distance coordinate for $j$ th liquid layer \\
\hline$\theta$ & circumferential angle \\
\hline$\lambda_{m}$ & $m$ th root of $J_{1}^{\prime}(\lambda)=0$ \\
\hline$\xi$ & $=r / R=$ dimensionless radial distance coordinate \\
\hline$\rho_{j}$ & mass density of $j$ th liquid layer \\
\hline$\phi_{j}$ & velocity potential function for $j$ th liquid layer \\
\hline$\psi_{j}$ & $\begin{array}{l}\text { velocity potential function associated with relative motion of liquid and } \\
\text { tank wall, given by Eq. (3) }\end{array}$ \\
\hline$\omega_{m n}$ & $\begin{array}{l}\text { circular natural frequency of layered system for } m \text { th horizontal and } n \text {th } \\
\text { vertical mode of vibration }\end{array}$ \\
\hline
\end{tabular}



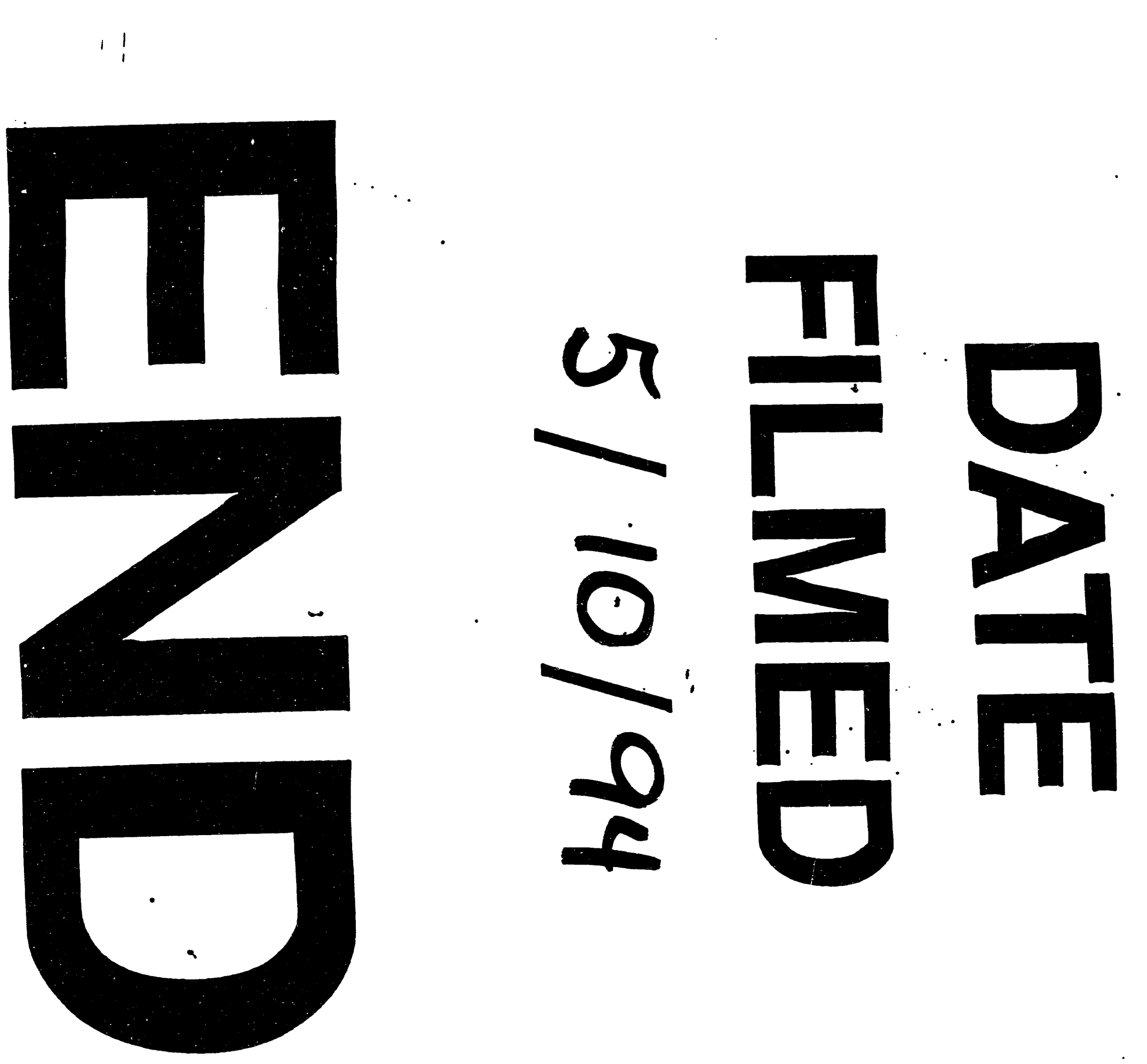
